Article

\title{
Asymptotic Safety: Swampland or Wonderland?
}

\author{
Ivano Basile $^{1}$ (D) and Alessia Platania ${ }^{2, *(D)}$ \\ 1 Service de Physique de l'Univers, Champs et Gravitation, Université de Mons, Place du Parc 20, \\ 7000 Mons, Belgium; ivano.basile@umons.ac.be \\ 2 Perimeter Institute for Theoretical Physics, 31 Caroline St. N., Waterloo, ON N2L 2Y5, Canada \\ * Correspondence: aplatania@perimeterinstitute.ca
}

check for

updates

Citation: Basile, I.; Platania, A. Asymptotic Safety: Swampland or Wonderland? Universe 2021, 7, 389. https://doi.org/10.3390/

universe7100389

Academic Editor: Antonio Pereira

Received: 14 September 2021

Accepted: 12 October 2021

Published: 18 October 2021

Publisher's Note: MDPI stays neutral with regard to jurisdictional claims in published maps and institutional affiliations.

Copyright: (C) 2021 by the authors. Licensee MDPI, Basel, Switzerland. This article is an open access article distributed under the terms and conditions of the Creative Commons Attribution (CC BY) license (https:// creativecommons.org/licenses/by/ $4.0 /)$.

\begin{abstract}
We investigate the consequences of combining swampland conjectures with the requirement of asymptotic safety. To this end, we explore the infrared regime of asymptotically safe gravity in the quadratic one-loop approximation, and we identify the hypersurface spanned by the endpoints of asymptotically safe renormalization group trajectories. These comprise the allowed values of higher-derivative couplings, as well as standard logarithmic form factors. We determine the intersection of this hypersurface with the regions of parameter space allowed by the weak-gravity conjecture, the swampland de Sitter conjecture, and the trans-Planckian censorship conjecture. The latter two depend on some order-one constants, for generic values of which we show that the overlap region is a proper subspace of the asymptotically safe hypersurface. Moreover, the latter lies inside the region allowed by the weak gravity conjecture assuming electromagnetic duality. Our results suggest a non-trivial interplay between the consistency conditions stemming from ultraviolet completeness of the renormalization group flow, black hole physics, and cosmology.
\end{abstract}

Keywords: asymptotic safety; quadratic gravity; swampland conjectures

\section{Introduction}

The 20th century has seen the development of two pillars of modern theoretical physics: quantum field theory (QFT) and general relativity (GR). The standard model of particle physics, which successfully describes the quantum properties of the strong and electroweak interactions, is based on the former framework. However, its naïve application to the latter yields a QFT of gravity prone to (perturbatively) non-renormalizable ultraviolet (UV) divergences [1-3].

Despite remarkable progress in a number of directions, the difficulties of formulating a complete theory of quantum gravity have led a considerable portion of the community to shift the focus on general, possibly model-independent lessons that could shed light on the nature of gravity at all scales. Many of these proposals, commonly dubbed "swampland conjectures" [4] in the context of string theory, rest on considerations on black-hole physics, which often can lie entirely in the semi-classical regime where one expects low-energy effective field theory (EFT) to be a reliable description.

On the other hand, some aspects of these conjectures arose from and are tied to string theory and, in particular, its spacetime-supersymmetric incarnations. These settings are much better understood, since quantum corrections are often under quantitative control [5-15] and can sometimes even be computed exactly [16,17]. In certain settings, such as $\mathcal{N}=2$ Calabi-Yau flux compactifications, one can even classify general families of models at once [18-23]. In some string models with high-energy supersymmetry breaking, a variety of swampland proposals were verified [24,25], although a construction of realistic and (meta-) stable vacua is still an open problem [26-57]. At any rate, it is paramount to understand the consequences of general consistency conditions outside of the specific contexts arising from (supersymmetric) string compactifications, although parametric control is most likely going to be problematic $[32,46,48,50,58]$ due to unknown or uncalculable 
corrections. To wit, efforts to test swampland proposals have almost entirely focused on supersymmetric settings, and in particular on stringy constructions. In order to shed light on whether they only encode a "string lamppost principle" [59] or they hold in more generality, it is important to extend their exploration to a broader class of quantum gravity models. On the other hand, the more stringent and well-grounded swampland proposals, such as the "no global symmetries" [60-63] and weak gravity [64] conjectures, could help guide the search for asymptotic safety, which is currently faced with the daunting prospect of navigating ever-larger theory spaces $[65,66]$.

A concrete problem that can be phenomenologically relevant in the near future is constraining the possible values of the Wilson coefficients of a curvature expansion of the gravitational EFT. In particular, one can expect that generic detectable leading-order effects of quantum gravity be encoded in the coefficients of the quadratic curvature invariants, which we shall discuss in detail in the following, or in some specific non-local form factors $[67,68]$. Some efforts in this direction have been made using the S-matrix bootstrap $[69,70]$, finding compelling agreement with the parameter space allowed by string theory. In the EFT framework, the problem has also been investigated via positivity bounds [71-80].

In this paper we approach this issue from a novel direction, studying the constraints coming from swampland conjectures together with the consistency conditions required by the existence of a UV fixed point of the gravitational renormalization group (RG) flow ${ }^{1}$. The latter scenario has been termed "asymptotic safety", in analogy with asymptotic freedom as a particular case. This idea, originally due to Weinberg [82], would imply that the Wilson coefficients of the IR effective action stem from a UV-complete RG trajectory, which in turn would be determined by a finite number of relevant deformations from the fixed point. Recently, this area of research has witnessed considerable development of the theoretical framework to investigate RG flows beyond perturbation theory [83] and finding evidence for the existence of the Reuter fixed point in a variety of different approximation schemes [66,84-113] (see also [114,115] for critical assessments on the status of the field and its open questions). Possible implications of asymptotically safe gravity in astrophysics and cosmology (see [116,117] for reviews) have been investigated using simplified models [118-140] and more elaborate computations [141], leading to the tentative conclusions that black-hole and cosmological singularities could be resolved by quantum effects, and that the nearly scale-invariant cosmological power spectrum could arise naturally from a nearly scale-invariant asymptotically safe regime.

In this paper we shall propose a concrete method to extract the allowed region of IR parameters from the RG flow of asymptotically safe trajectories. In particular, we shall focus on the simpler case of the one-loop approximation in quadratic gravity $[87,142,143]$ in order to test our construction and provide a proof of principle of our idea. We will show that the IR limit of asymptotically safe trajectories falls inside the region allowed by the weak gravity conjecture and electromagnetic duality, and display a non-trivial intersection with the one allowed by the de Sitter and trans-Planckian censorship bounds.

The contents of this paper are organized as follows. In Section 2 we provide a brief overview of swampland conjectures, focusing on the weak gravity conjecture, the de Sitter conjecture and the trans-Planckian censorship conjecture, since they entail the most relevant bounds for our subsequent analysis. In Section 3 we describe in detail the one-loop approximation to quadratic gravity that we employ as testing grounds, and our method of extracting the physical IR Wilson coefficients. The resulting effective action turns out to contain non-local form factors. In Section 4 we collect our results: in Section 4.1 we present the allowed region of parameter space that we found, which spans a plane in the three-dimensional space of dimensionless IR parameters, and in Sections 4.2 and 4.3 we study the constraints stemming from the swampland conjectures discussed in Sections 2.1 and 2.2 respectively. In Section 4.4 we discuss and display the intersection of all regions. We conclude with a summary and some perspectives in Section 5. 


\section{An Overview of Swampland Conjectures}

As we have anticipated in the introduction, swampland conjectures are proposals that ought to rule out EFTs of gravity that do not admit UV completions [4]. These conjectures are generally motivated in part by purely low-energy considerations, stemming from black-hole physics or inflation, but they also arise from detailed investigations of stringtheoretic settings, where generally one has more control over corrections and patterns can be corroborated across families of EFTs. The latter approach has led some to describe a "lamppost" effect [59], whereby only settings that are somewhat under control can be investigated and thus it is unclear to which extent the resulting conclusions can be generalized. Furthermore, while at least minimal supersymmetry is generally unbroken in order to retain computational control, recent considerations $[144,145]$ point to a tension between low-energy supersymmetry breaking ${ }^{2}$ and the consistency of the EFT. As we have discussed in the preceding section, one of the motivations behind this work is indeed to go beyond the usual settings, seeking lessons for other approaches to quantum gravity.

Since its inception, the swampland program aims to describe the boundary between the landscape of consistent gravitational EFTs with a growing number of proposed crite$\mathrm{ria}^{3}$, numerous relations among which $[152,153]$ point to a deeper underlying principle. In particular, connections between the distance conjecture $[154,155]$ and string dualities suggest that an organizing principle for these consistency criteria in the IR be related to a non-perturbative UV formulation of quantum gravity. Furthermore, as we shall see in the following, swampland considerations have provided intriguing clues toward a number of phenomenological puzzles [156].

In this paper we shall focus on some conjectures which can provide bounds for the Wilson coefficients of the gravitational EFT. In particular,

- The weak gravity conjecture (WGC) [64] relates the mass and charge of light states and black holes;

- The de Sitter conjecture (dSC) [157], along with its refined versions [155,158,159], constrains the behavior of scalar potentials and their derivatives, leading to an obstruction to the existence of de Sitter vacua that is $\mathcal{O}(1)$ in Planck units;

- The trans-Planckian censorship conjecture (TCC) $[160,161]$ constrains sub-Planckian cosmological perturbations to remain sub-Planckian across inflation, and leads to bounds on the lifetime of metastable de Sitter configurations as well as on the $\mathcal{O}(1)$ parameter that appears in the $\mathrm{dSC}$, at least in asymptotic regions of field space.

In light of the latter consideration, for the purposes of this paper in the following we shall investigate the consequences of the TCC on Starobinsky-like inflationary potentials as a special case of the dSC. Indeed, we shall restrict ourselves to the asymptotic region of field space corresponding to small curvatures in Planck units, where the TCC could provide a dSC bound with a specific $\mathcal{O}(1)$, as we shall see below.

\subsection{Weak Gravity Conjecture and Black Holes}

Let us begin reviewing some features of the (electric) WGC, referring the reader to $[150,151]$ for more details. In its most basic form, it states that in a consistent EFT of gravity coupled to a $U(1)$ gauge field there exists a state whose mass $m$ is lower than its charge $q$ in Planck units. In four dimensions, the bound for charged particles reads

$$
\frac{m}{M_{\mathrm{Pl}}} \leq \mathcal{O}(1) q,
$$

where the model-dependent $\mathcal{O}(1)$ constant is $\frac{1}{\sqrt{2}}$ in Einstein-Maxwell theory.

Among various motivations and evidence gathered in the literature, the WGC is grounded in black-hole physics from the requirement that charged, extremal black holes be able to decay, lest protected by a symmetry (such as supersymmetry, in the case of BPS-saturated states). The rationale behind this lies in avoiding remnants while keeping the black hole from violating the extremality bound, since a violation of either would 
presumably lead to consistency issues potentially within the EFT regime $[64,162,163]$. For charged black holes of mass $M$ and charge $Q$, this requirement translates into

$$
\frac{M}{Q} \geq\left(\frac{M}{Q}\right)_{\text {extremal }}
$$

where the latter is generally an $\mathcal{O}(1)$ constant $^{4}$. However, higher-curvature corrections could potentially spoil this condition even for macroscopic black holes, provided they are sufficiently close to extremality. Writing the leading quartic corrections according to [164]

$$
\Delta \mathcal{L}=c_{1} R^{2}+c_{2} R_{\mu v} R^{\mu v}+c_{3} R_{\mu v \rho \sigma} R^{\mu v \rho \sigma},
$$

the resulting bounds for the corresponding Wilson coefficients $c_{i}$ comprise a family of inequalities for linear combinations of the $c_{i}$, parametrized by the extremality parameter of the black hole $[64,164-168]$. The extremality bound in general now takes the form

$$
\frac{M}{Q} \geq\left(\frac{M}{Q}\right)_{\text {extremal }}\left(1-\frac{\Delta}{M^{2}}\right)
$$

where the linear combination $\Delta$ of Wilson coefficients is to be non-negative in order for the WGC to hold, and is proportional to the coefficient $c_{2}+4 c_{3}$ of the Weyl-squared term $[164,168]$.

The leading order contributions to $\Delta$ comprise not only the Wilson coefficients in the effective action of Equation (3), but also the Wilson coefficients that involve the $U(1)$ gauge field. It has been recently shown [169] that, assuming invariance under electromagnetic duality, higher-curvature corrections up to sextic order can be written in terms of purely gravitational terms, up to field redefinitions. Let us stress that our aim is to intersect swampland bounds with the constraints provided by asymptotic safety, and the technical obstacles to compute its consequences for quartic electromagnetic couplings in gravity, which would entail involved FRG computations along the lines of [66], compel us to focus on the duality-invariant scenario of [169], which at any rate appears intriguing on its own ${ }^{5}$. Moreover, the electromagnetic couplings do not run under the RG flow at one loop because of tree-level duality $[166,168]$. This has been used to argue that the low-energy behavior of the correction $\Delta$ to the extremality bound is dominated by the Weyl anomaly coefficients that drive the running of the $c_{i}$, and in particular of the Weyl-squared Wilson coefficient $c_{2}+4 c_{3}$, as we shall see in the following. However, in the present case we would like to constrain the physical parameters built out of the Wilson coefficients and the Planck scale, in order to compare the resulting bounds with the constraints of asymptotic safety. We shall describe the procedure in detail in the following section.

\section{2. de Sitter and Trans-Planckian Censorship}

Let us now move on to discuss the dSC and the TCC. The former quantifies an obstruction to the existence of de Sitter vacua, in the form of a bound for the (field-space gradient of the) scalar potential $V(\phi)$. Indeed, since in this setting de Sitter vacua would arise as positive-energy critical points of $V$, a natural bound that would prevent these takes the form

for field ranges

$$
M_{\mathrm{Pl}}|\nabla V| \geq c V
$$

$$
\Delta \phi \lesssim f M_{\mathrm{Pl}}
$$

where $c, f>0$ are (a priori model-dependent) constants. Within the EFT framework, their natural values are $\mathcal{O}(1)$, indicating that the obstruction is tied to the expected cutoff of the EFT. However, one is readily confronted with a tension between the bound in Equation (5) and slow-roll inflation $[158,171]^{6}$, leading to refinements involving the Hessian matrix 
of the potential $[155,158]$. In particular, whenever the bound of Equation (5) would be violated, the matrix

$$
M_{\mathrm{Pl}}^{2} \operatorname{Hess}(V)+c^{\prime} V
$$

would be negative semidefinite, with $c^{\prime}>0$ another $\mathcal{O}(1)$ constant. Further refinements were proposed in [159], but in our setting we shall find that the first bound of Equation (5) is sufficient, since it encompasses Equation (7) in the regions of parameter space that we are concerned with.

On the other hand, the TCC surmises that sub-Planckian quantum fluctuations in the early universe at initial time $t_{i}$ never grow macroscopic at a final time $t_{f}$. In particular, they ought to never cross the Hubble horizon and freeze. This requirement can be formulated, in terms of the scale factor $a(t)$ and the corresponding Hubble parameter $H$, by $[160,161]$

$$
\frac{a\left(t_{f}\right)}{a\left(t_{i}\right)} \lesssim \frac{M_{\mathrm{Pl}}}{H\left(t_{f}\right)}
$$

again up to an $\mathcal{O}(1)$ constant. An intriguing consequence of Equation (8) is that de Sitter configurations are not prohibited, but they are metastable with a lifetime $T$ bounded by

$$
T \lesssim \frac{1}{H} \log \frac{M_{\mathrm{Pl}}}{H}
$$

of the order of a trillion years. This results points to a possible resolution of the coincidence problem in this setting.

The most relevant consequence of the TCC for the purposes of this paper is that, in the presence of a scalar potential, it leads to a bound of the form of Equation (5) with

$$
c=\frac{2}{\sqrt{(d-1)(d-2)}}
$$

in $d$ spacetime dimensions, at least in asymptotic regimes of field space. In the present setting, the scalar potential arises from the quadratic curvature terms, and the corresponding asymptotic regime for gravitational field fluctuations is that of small curvatures [176]. This regime is mapped to a neighbourhood of the origin in the inflaton description. For generic curvatures, one expects that both the purely gravitational description and the inflaton description be modified, including the geometry of field space. Nevertheless, since our current setup does not allow for precise quantitative bounds, we shall henceforth take Equation (10) simply as a reference point around which to study the more general bound of Equation (5). Let us also remark that this value appears in a number of related swampland bounds [152] and is well-behaved under dimensional reduction [172], and thus it may play a more prominent role in the story. At any rate, it would be interesting to explore the more direct implications of Equation (8) studying cosmological solutions or exploring the considerations of $[161,177]$ within our setup.

\section{One-Loop RG Flow in Quadratic Gravity}

Let us now discuss the concrete setting in which we shall compute the possible values of the Wilson coefficients of the effective gravitational action. In this work we focus on the quadratic truncation ${ }^{7}$, in the one-loop approximation. In Euclidean signature, the Lagrangian pertaining to the full quadratic truncation reads

$$
\mathcal{L}=\frac{2 \Lambda-R}{16 \pi G}+\frac{1}{2 \lambda} C^{2}-\frac{\omega}{3 \lambda} R^{2}+\frac{\theta}{\lambda} E,
$$

where $C^{2} \equiv C_{\mu v \rho \sigma} C^{\mu v \rho \sigma}$ is the square of the Weyl tensor, $E$ is the Gauss-Bonnet density and and the Wilson coefficients

$$
g_{C} \equiv \frac{1}{2 \lambda}, \quad g_{R} \equiv-\frac{\omega}{3 \lambda}
$$


can be related to the $c_{i}$ coefficients in Equation (3). Indeed, since

$$
\begin{gathered}
C^{2}=R_{\mu v \rho \sigma} R^{\mu v \rho \sigma}-2 R_{\mu v} R^{\mu v}+\frac{R^{2}}{3}, \\
E=R_{\mu \nu \rho \sigma} R^{\mu v \rho \sigma}-4 R_{\mu v} R^{\mu v}+R^{2},
\end{gathered}
$$

the $c_{i}$ are related to the couplings in Equation (11) according to

$$
\begin{aligned}
& c_{1}=\frac{1}{6 \lambda}-\frac{\omega}{3 \lambda}+\frac{\theta}{\lambda}, \\
& c_{2}=-\frac{1}{\lambda}-\frac{4 \theta}{\lambda}, \\
& c_{3}=\frac{1}{2 \lambda}+\frac{\theta}{\lambda} .
\end{aligned}
$$

While this setup holds in general spacetime dimensions $d$, we now restrict to $d=4$. The one-loop beta functions of the couplings of Equation (11) read [87,142,143]

$$
\begin{aligned}
\beta_{\widetilde{\Lambda}}= & -2 \widetilde{\Lambda}+\frac{1}{(4 \pi)^{2}}\left[\frac{1+20 \omega^{2}}{256 \pi \widetilde{G} \omega^{2}} \lambda^{2}+\frac{1+86 \omega+40 \omega^{2}}{12 \omega} \lambda \widetilde{\Lambda}\right] \\
& -\frac{1+10 \omega^{2}}{64 \pi^{2} \omega} \lambda+\frac{2 \widetilde{G}}{\pi}-\frac{83+70 \omega+8 \omega^{2}}{18 \pi} \widetilde{G} \widetilde{\Lambda}, \\
\beta_{\widetilde{G}}= & 2 \widetilde{G}-\frac{1}{(4 \pi)^{2}} \frac{3+26 \omega-40 \omega^{2}}{12 \omega} \lambda \widetilde{G}-\frac{83+70 \omega+8 \omega^{2}}{18 \pi} \widetilde{G}^{2}, \\
\beta_{\lambda}= & -\frac{1}{(4 \pi)^{2}} \frac{133}{10} \lambda^{2}, \\
\beta_{\omega}= & -\frac{1}{(4 \pi)^{2}} \frac{25+1098 \omega+200 \omega^{2}}{60} \lambda, \\
\beta_{\theta}= & \frac{1}{(4 \pi)^{2}} \frac{7(56-171 \theta)}{90} \lambda
\end{aligned}
$$

where $\widetilde{G}_{k}=G_{k} k^{2}$ and $\widetilde{\Lambda}_{k}=\Lambda_{k} k^{-2}$ are the dimensionless Newton coupling and cosmological constant respectively, and we have suppressed the subscript $k$ in Equation (15) for the sake of clarity.

In our setting, the flow of the (classically) marginal couplings $\lambda, \omega$ and $\theta$ is decoupled from that of the Einstein-Hilbert couplings. Out of the UV fixed points

$$
\lambda_{*}=0, \quad \omega_{*}=\omega_{ \pm} \equiv \frac{-549 \pm 7 \sqrt{6049}}{200}, \quad \theta_{*}=\frac{56}{171},
$$

UV completeness selects $\omega_{*}=\omega_{+} \approx-0.023$ [178], which the solutions approach as the RG time $^{8} t \equiv \log \frac{k_{0}}{k} \rightarrow-\infty$, as is apparent from Figure 1. Let us remark that this fixed point is asymptotically safe, i.e., at least one coupling is not asymptotically free $[142,143,178,179]$. Indeed, the critical exponents of suitable linear combinations of $G$ and $\Lambda$ are 2 and $4^{9}$, while in the IR they become the canonical -2 and 2 respectively [181]. The fact that all couplings are attracted to the fixed point in the UV [178] is instead an artifact of the oneloop approximation. Indeed, more sophisticated FRG computations yield a fixed point with a three-dimensional critical surface [89]. 


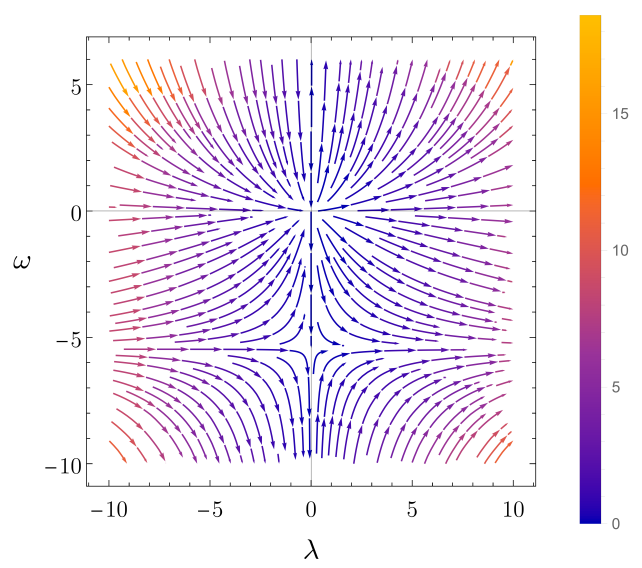

Figure 1. Flow of one-loop quadratic gravity in the $(\omega, \lambda)$ subspace. The arrows point toward the IR. In this setting, two non-trivial fixed points are present, and the Reuter fixed point is the one with the smaller absolute value [178].

The flow can be solved analytically in terms of the deformations $\delta \lambda, \delta \omega$ from the UV fixed point, and yields the closed-form solution

$$
\begin{aligned}
& \lambda(t)=\frac{\delta \lambda}{1-\frac{133}{160 \pi^{2}} \delta \lambda t}, \\
& \omega(t)=\frac{\omega_{-}-\omega_{+}\left(1+\frac{\Delta}{\delta \omega}\right)\left(1-\frac{133}{160 \pi^{2}} \delta \lambda t\right)^{\frac{7 \sqrt{6049}}{399}}}{1-\left(1+\frac{\Delta}{\delta \omega}\right)\left(1-\frac{133}{160 \pi^{2}} \delta \lambda t\right)^{\frac{7 \sqrt{6049}}{399}}}, \\
& \theta(t)=\frac{56}{171}+\frac{\delta \theta}{1-\frac{133}{160 \pi^{2}} \delta \lambda t},
\end{aligned}
$$

where $\Delta \equiv \omega_{+}-\omega_{-}$. The vector field generating this flow is displayed in Figure 1 in the $(\omega, \lambda)$ subspace and in Figure 2 with various 3D plots. Let us observe that the UV completeness of the trajectory requires $\delta \lambda>0$, and that the IR flow ends at $t=t_{\mathrm{IR}} \equiv \frac{160 \pi^{2}}{133 \delta \lambda}$. However, since $\delta \lambda \ll 1$ this RG time is parametrically large, and one can reliably extract the perturbative IR behavior for the Wilson coefficients. Furthermore, reaching a physical IR regime with $\widetilde{G} \rightarrow 0^{+}$requires that $\delta \widetilde{G}, \delta \omega<0$, in order that their flows remain between the UV and IR fixed-point values avoiding runaway. The flow of the relevant deformations from the fixed point is shown in Figure 3. 

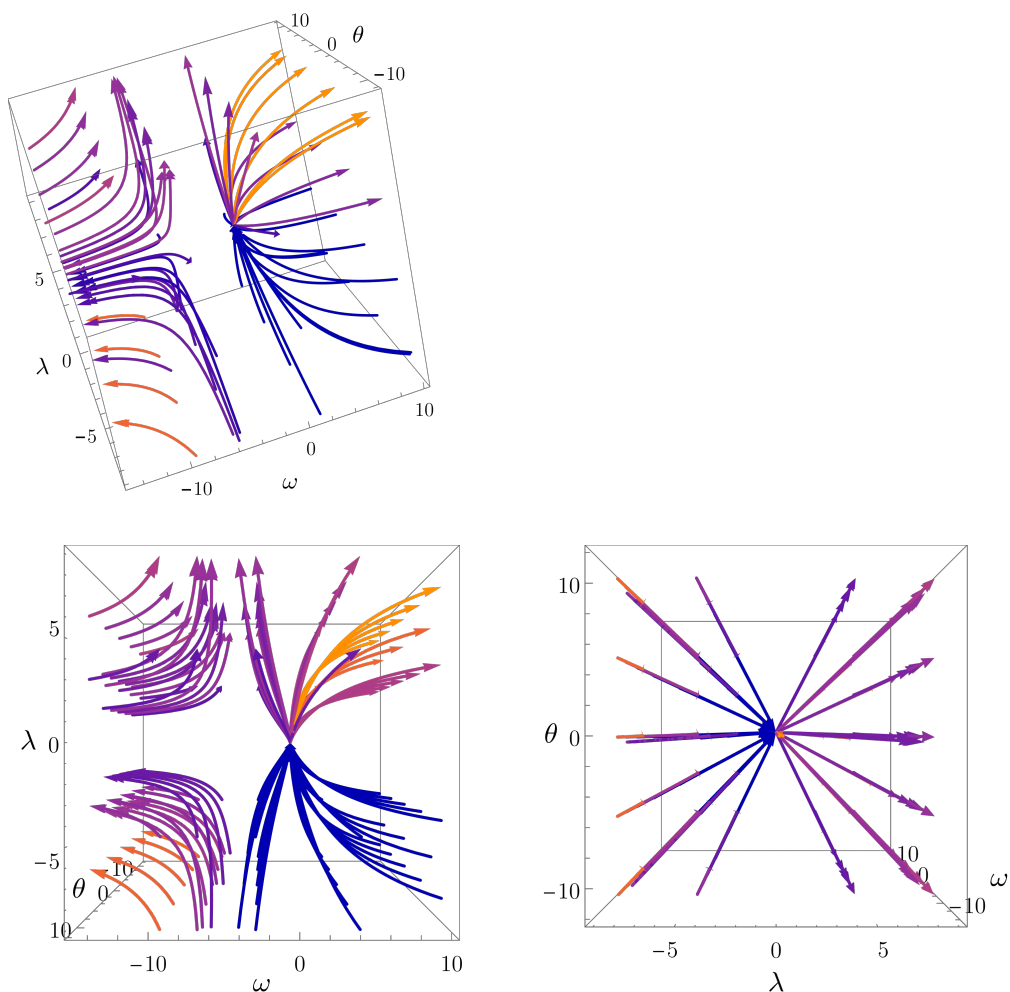

Figure 2. Flow of the classically marginal couplings $(\omega, \lambda, \theta)$ in one-loop quadratic gravity. The arrows point towards the IR, and different viewpoints are shown to better visualize the flow. The color coding of the arrows is identical to that of Figure 1.
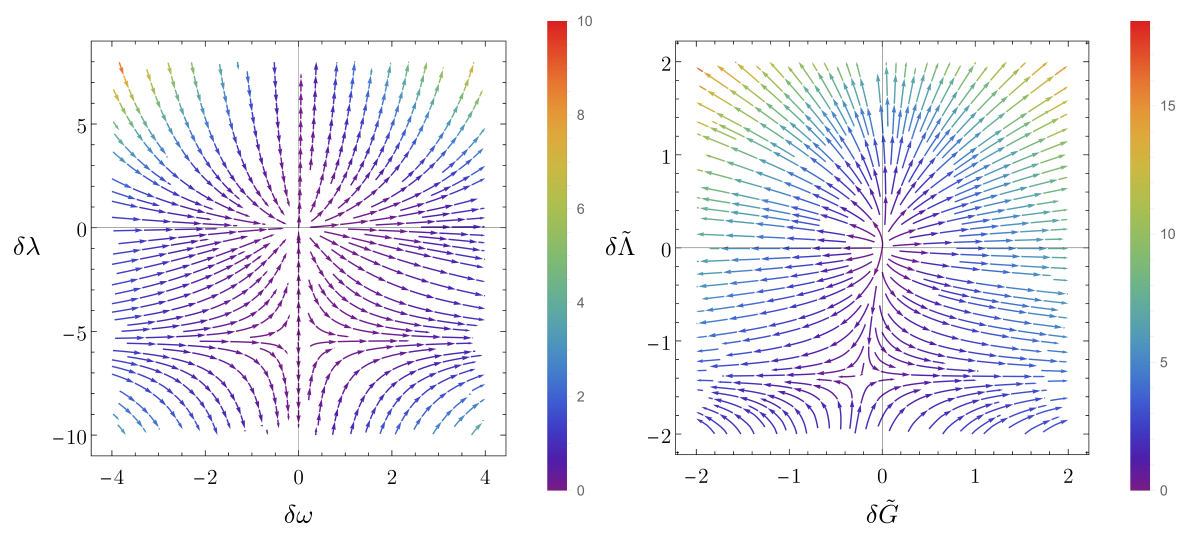

Figure 3. Flow of the relevant deformations from the fixed point. The left panel depicts the flow in the $(\omega, \lambda)$ subspace. The right panel depicts the flow in the $(G, \Lambda)$ subspace where the classically marginal couplings have been set to the UV fixed point. The arrows indicate the the flow from the UV to the IR.

Substituting the expressions of Equation (17) in Equation (15), one can then solve the remaining flow equations numerically varying the initial conditions, or, equivalently, with a random sampling of the deformations $\delta \omega, \delta \lambda, \delta \widetilde{G}, \delta \widetilde{\Lambda}$ from the UV fixed point. The RG flow then drives the running couplings to the weakly coupled IR, where the running couplings $g_{C}$ and $g_{R}$, defined in Equation (12), behave logarithmically (linearly in $t$ ) as $t \rightarrow t_{\mathrm{IR}}^{-}$ This result is consistent with perturbative computations, and the resulting asymptotic expressions read 


$$
\begin{aligned}
& g_{C}(t) \sim \frac{1}{2 \delta \lambda}-\frac{133}{320 \pi^{2}} t \\
& g_{R}(t) \sim-\frac{\omega_{-}}{3 \delta \lambda}+\frac{133}{480 \pi^{2}} \omega_{-} t .
\end{aligned}
$$

In order to extract the physical IR parameters, we shall identify the (square of the) RG scale $k^{2}$ with the covariant Laplacian/d'Alembertian $\square$. In order to eliminate the arbitrary reference scale $k_{0}$ that defines the initial condition for the RG flow, one can express every quantity in units of the IR Planck mass ${ }^{10} M_{\mathrm{Pl}}^{-2}=G$. To this end, since $e^{2 t} \widetilde{G}(t) \rightarrow \widetilde{G}_{0}$ tends to a constant in the IR, one can evaluate the running Wilson coefficients of Equation (18) replacing $t \rightarrow-\frac{1}{2} \log \widetilde{G}(t)$, so that

$$
\begin{aligned}
\log \frac{M_{\mathrm{Pl}}}{k} & =\log \frac{M_{\mathrm{Pl}}}{k_{0}}+t \\
& \sim-\frac{1}{2} \log \widetilde{G}(t) \\
& \sim-\frac{1}{2} \log \widetilde{G}_{0}+t .
\end{aligned}
$$

Since $\widetilde{G}_{0}$ can be extracted from the numerical solution of Equations (15), identifying

$$
\log \frac{k^{2}}{M_{\mathrm{Pl}}^{2}} \longrightarrow \log \frac{\square}{M_{\mathrm{Pl}}^{2}}
$$

according to the preceding considerations, one can reconstruct an effective action of the form

$$
\Gamma=\int d^{4} x \sqrt{g}\left(\frac{2 \Lambda-R}{16 \pi G}+g_{C} C^{2}+g_{R} R^{2}+b_{C} C \log \frac{\square}{M_{\mathrm{Pl}}^{2}} C+b_{R} R \log \frac{\square}{M_{\mathrm{Pl}}^{2}} R\right),
$$

where Weyl-tensor contraction is understood. The appearance of non-local form factors resonates with the considerations in [182-184]. While we shall neglect them in the following, the presence of form factors of this type seems largely consistent with preceding results [185-190] (see also [191] for a discussion of logarithmic form factors). Note that, despite their behavior at low energies, one expects a resummation of such non-local form factors to yield a result that is both well-defined and subleading in the IR compared to the local terms $[183]^{11}$. Furthermore, they do not contribute to the scalar potential that we shall discuss in Section 4.3 in the context of the dSC. Notwithstanding the importance of form factors in establishing a non-local behavior of gravity, we would like to understand which values of the three dimensionless combinations

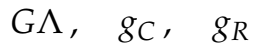

are allowed starting from any initial condition, i.e., any perturbation of the asymptotically safe UV fixed point along UV-attractive directions. To this end, we have evaluated numerically these combinations in the IR, implementing the substitution of Equation (19). The following plots highlighting the swampland constraints, the IR limits of asymptotically safe RG trajectories, as well as the final intersection between the allowed regions, will pertain to the $\left(G \Lambda, g_{C}, g_{R}\right)$ theory space.

To conclude this section, let us collect a few words of caution regarding the one-loop approximation. In general, in the context of gravity, one expects it to be only reliable in the IR, despite the appearance of a UV fixed point outside of the perturbative regime. The methods of the functional RG have been employed, both in earlier $[89,192]$ and recent $[66]$ efforts, to obtain non-perturbative flow equations in the quadratic truncation, but applying our method to extract the allowed region of parameter space in the IR entails highly involved and unstable numerical analysis. In order to circumvent these obstacles, and address the 
problem in a more quantitative fashion, a natural first step would entail performing novel FRG computations. The simplest relevant setting would include the most general quadratic truncation coupling the electromagnetic field to gravity, which, while daunting, appears feasible via the methods that have been very recently introduced in [66] to study the purely gravitational sector. In light of these (and other related) issues, in this work we have focused on the one-loop approximation as a proof of principle, with the hope of uncovering some instructive general lessons from the results that we are now about to present.

Finally, let us stress that, although quadratic truncations of the gravitational action are typically associated with a loss of physical unitarity [193], the Stelle ghost could be a truncation artifact [194]. Integrating out quantum fluctuations could lead to well-behaved, unitary scattering amplitudes [183,184], as explicit computations seem to suggest [195]. This issue was also discussed within the setting explored in this paper in [142,143].

\section{Results}

Let us now describe in detail our results on the allowed values of physical IR parameters that we have obtained from the calculations outlined in the preceding section, along with the swampland constraints that we have discussed in Section 2.

\subsection{Infrared Limit of Asymptotically Safe RG Trajectories in One-Loop Quadratic Gravity}

In order to uncover the space of physical parameters appearing in the (local sector of the) effective action of Equation (21), we have sampled the space of allowed deformations $(\delta \omega, \delta \lambda, \delta \widetilde{G}, \delta \widetilde{\Lambda})$ from the UV fixed point, and extracted the resulting IR values of the parameters in Equation (22) evaluating the flow of $\widetilde{G}$ and $\widetilde{\Lambda}$ for a suitably large RG time $t \approx 30$, exploiting the rapid convergence of the combination in Equation (19). The resulting values for $\widetilde{G} \widetilde{\Lambda}=G \Lambda$, or equivalently $\Lambda / M_{\mathrm{Pl}}^{2}$, span a wide range of values, of the order of $10^{5}$ for the region of initial deformations that we have explored. Moreover, the closed-form flow that one obtains setting the classically marginal couplings to their fixed-point values spans the whole real axis [178]. We are thus led to conclude that the allowed (IR) values of $G \Lambda$ are unrestricted. On the other hand, the values of $g_{C}$ and $g_{R}$ appear to lie on the line

$$
g_{R} \approx-0.74655+3.64447 g_{C},
$$

as depicted in Figures 4 and 5. This result appears to be very robust upon increase of the sample size, and in particular for $10^{6}$ points the covariance matrix of the fit is of the order $\mathcal{O}\left(10^{-8}\right)$. Let us observe that, neglecting the intercept term, Equation (23) follows from Equation (18) as $t \rightarrow t_{\mathrm{IR}}^{-}$, whereby $g_{R} \sim-2 \omega_{-} / 3 g_{C}$. Since we instead evaluate the IR couplings at a fixed, albeit sufficiently large, $R G$ time, it is tempting to speculate that the intercept term in Equation (23) is a correction arising from RG trajectories that approach the IR more slowly. Therefore, at least within the scope of our approximations, the presence of a UV fixed point appears to constrain the allowed physical coefficients in Equation (22) to a specific plane, and we shall now compare this result to the constraints arising from the swampland conjectures that we have discussed in Section 2.

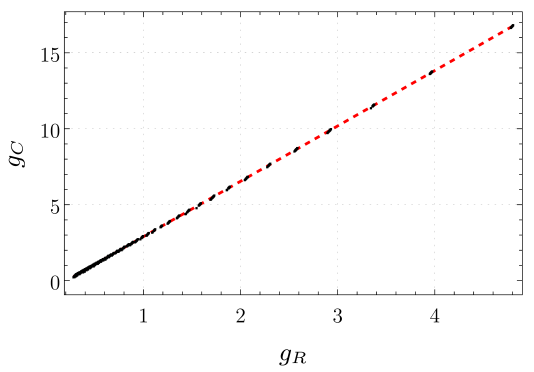

Figure 4. The line of equation $g_{R}=-0.74655+3.64447 g_{C}$ fitting the IR values obtained from the flow of Equation (15) sampling UV initial conditions. The covariance matrix evaluates to $\mathcal{O}\left(10^{-8}\right)$ with $10^{6}$ data points. 


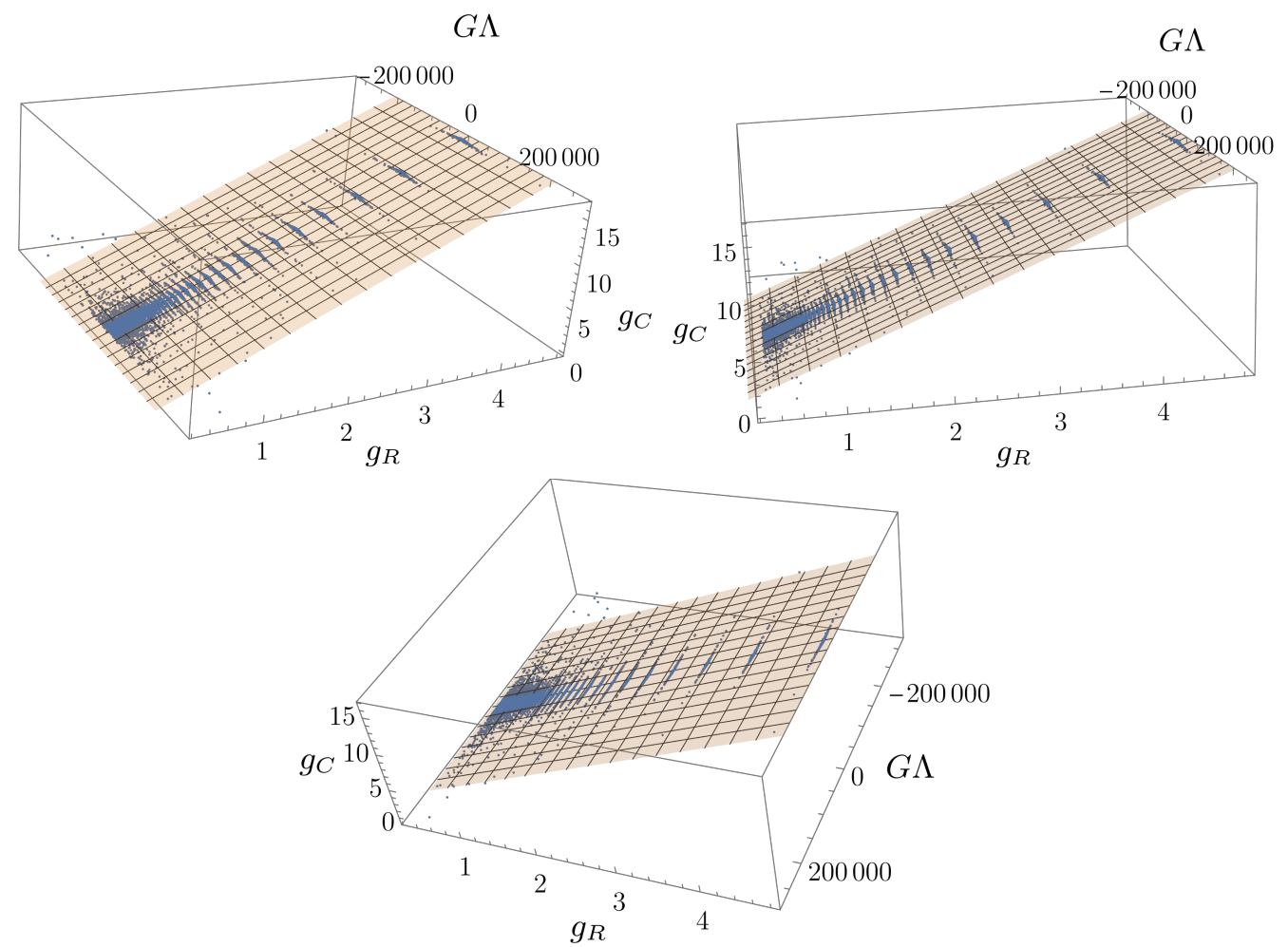

Figure 5. Plots depicting the IR endpoints of asymptotically safe RG trajectories. The points lie on the plane of equation $g_{R}=-0.74655+3.64447 g_{C}$. The values of $G \Lambda$ span a vast range, which, together with the closed-form flow of [178] depicted in Figure 3, leads us to infer that they are unrestricted.

\subsection{Constraints on Quadratic Gravity from WGC}

As we have discussed in Section 2.1, the WGC entails positivity bounds for the Wilson coefficients of the higher-derivative corrections to Einstein gravity. Since these bounds involve charged particles and black holes, higher-derivative couplings of a $U(1)$ gauge field ought to be included, although the resulting RG flow is extremely involved technically and has not been computed hitherto. On the other hand, the considerations of $[166,168,169]$, based on electromagnetic duality, show that one can still make use of our results to constrain higher-derivative corrections in a duality-invariant scenario using the WGC. To this end, expressing the higher-curvature in terms of the $c_{i}$ coefficients of Equation (3), the (family of) positivity bound(s) of [165] reads

$$
(1-\xi)^{2} c_{0}+20 \xi c_{3}-5 \xi(1-\xi)\left(2 c_{3}\right)>0
$$

where $\xi \equiv \sqrt{1-Q^{2} / M^{2}}$ is the extremality parameter of Reissner-Nordström black holes with mass $M$ and charge $Q, 0<\xi<1 / 2$ for black holes with positive specific heat and

$$
c_{0} \equiv c_{2}+4 c_{3} .
$$

In terms of the couplings in Equation (11), the bound of Equation (24) takes the simpler form

$$
\frac{1}{\lambda}\left(10 \theta(\xi+1) \xi+6 \xi^{2}+3 \xi+1\right)>0,
$$

which holds for $\lambda>0$ provided that $\xi>0$ (which is always satisfied by the extremality parameter) and that $\theta>0$. As we have discussed in Section 3, the latter condition is fulfilled if if $\delta \theta>0$, since $\delta \lambda>0$ in Equation (17). Hence, the (duality-invariant) WGC constrains $\lambda>0$, which is included by the analysis of the preceding section and does not entail additional conditions. Let us observe that, although $\theta$ encodes the coupling of the Gauss-Bonnet invariant, it contributes to the entropy of a black hole [196-198] even in four 
dimensions, where it is topological. It does not contribute in the limit $\xi \ll 1$, since the resulting bound also describes the positivity of the extremality ratio $[164,168]$.

\subsection{Constraints on Quadratic Gravity from $d S$ and TC Conjectures}

Let us now discuss the constraints arising from the dSC and the TCC. As we have anticipated in Section 2.2, we shall focus on the bounds that the dSC and the TCC entail for the scalar potential that arises from the higher-derivative corrections of Equation (21). In order to extract the potential proper, we shall concern ourselves with the local sector of the theory, neglecting the form factors and the Weyl term, which vanishes on cosmological backgrounds.

Following the standard procedure to obtain inflaton potentials from $F(R)$ Lagrangians (see, e.g., $[137,199])$, one begins from $R^{2}$ gravity with a cosmological constant,

$$
F(R)=\frac{1}{16 \pi G}\left(R-2 \Lambda+\frac{R^{2}}{6 m^{2}}\right),
$$

where the coupling $g_{R}$ is related to the inflaton mass according to

$$
g_{R}=-\frac{M_{\mathrm{Pl}}^{2}}{(8 \pi) \cdot 12 m^{2}} .
$$

One then arrives at the inflaton potential

$$
V(\phi)=\frac{M_{\mathrm{Pl}}^{2}}{8 \pi} e^{-2 \sqrt{\frac{2}{3}} \frac{\phi}{M_{\mathrm{Pl}}}}\left(\frac{3 m^{2}}{4}\left(e^{\sqrt{\frac{2}{3}} \frac{\phi}{M_{\mathrm{Pl}}}}-1\right)^{2}+\Lambda\right) .
$$

In order to retain compatibility with the EFT, we shall consider field excursions in Equation (6) around $\phi \ll M_{\mathrm{Pl}}$, since it corresponds to small curvatures. Indeed, the procedure to obtain inflationary potentials from quadratic gravity yields $\phi=\sqrt{\frac{3}{2}} M_{\mathrm{Pl}} \log \left(1+\mathcal{O}\left(M_{\mathrm{Pl}}^{-2}\right)\right)[137,199]$. One can then study dSC and TCC constraints of Equations (5), (7) and (8) numerically varying the $\mathcal{O}(1)$ constants $c$ and $f$, imposing that the bounds be satisfied for all $\phi$ in the range allowed by Equation (6). The resulting regions are highlighted in Figure 6 and in Figure 7, where each panel corresponds to a particular value of $f$ and consists of two plots which display the bounds in the $\left(m^{2}, \Lambda\right)$ (left panels) and $\left(g_{R}, G \Lambda\right)$ (right panels) planes. Due to the inverse relation in Equation (28) between $\mathrm{m}^{2} / M_{\mathrm{Pl}}^{2}$ and $g_{R}$, the linear bounds in the $\left(m^{2}, \Lambda\right)$ plane translate into hyperbolas in the $\left(g_{R}, G \Lambda\right)$ plane. Note that whether the dimensionless minimum $\phi_{\min } / M_{\mathrm{Pl}}$, which exists for $\Lambda / m^{2}>-3 / 4$, falls inside the interval $(-f,+f)$ depends on the ratio $\Lambda / m^{2}$. In particular, the minimum falls outside the interval for $f<\left|\phi_{\min } / M_{\mathrm{Pl}}\right|$. Consequently, even if $V\left(\phi_{\min }\right)$ were to be positive for some $\Lambda$ and $m^{2}$, this would not necessarily violate the dSC/TCC bounds for fixed values of $f$ and $c$. Consequently, the bounds displayed in Figure 6 and in Figure 7 are non-trivially affected by Equation (6), and by the specific values of $f$ and $c$. For instance, it is worth noticing that smaller values of $f$ entail smaller field intervals where the ASC/TCC bounds are to be satisfied. Thus, the bounds are less stringent, and the allowed region bigger. Similarly, the bound is more restrictive for higher values of $c$. In particular, Figure 7 depicts the bounds derived from $c=\sqrt{2 / 3}$, the value pertaining to the TCC. While our analysis cannot probe the TCC in the large-excursion regime, where it differs substantially from the dSC, the additional considerations of $[152,172]$ point to a deeper role of this value of $c$ which could manifest itself, in the low-curvature regime at stake, in further investigations of swampland bounds and/or dimensional reduction. 

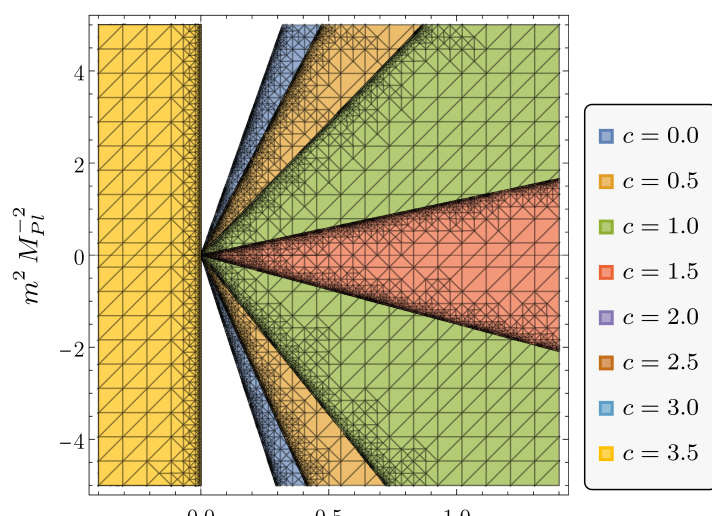

$\Lambda M_{P l}^{-2}$

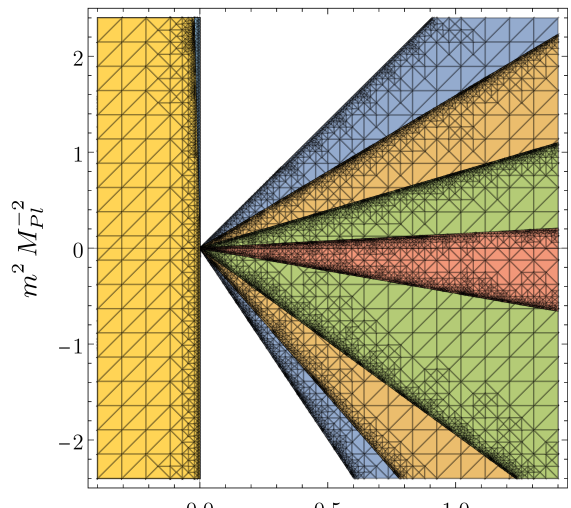

$M_{P l}^{-2}$

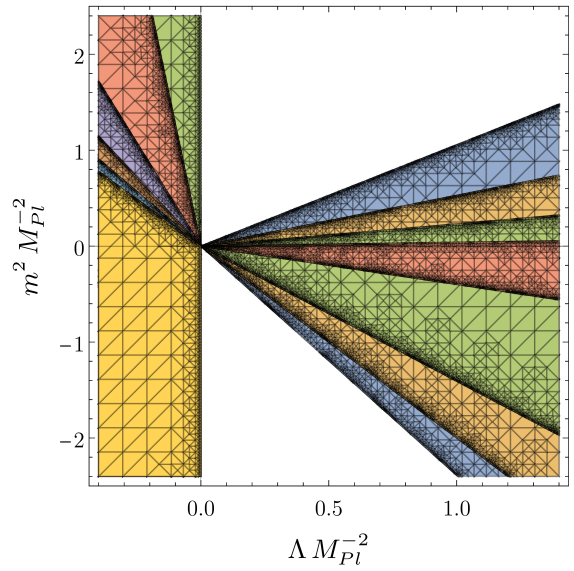

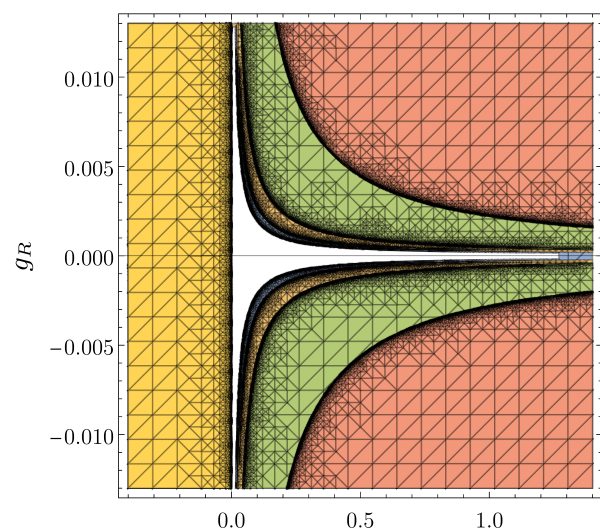

$G \Lambda$

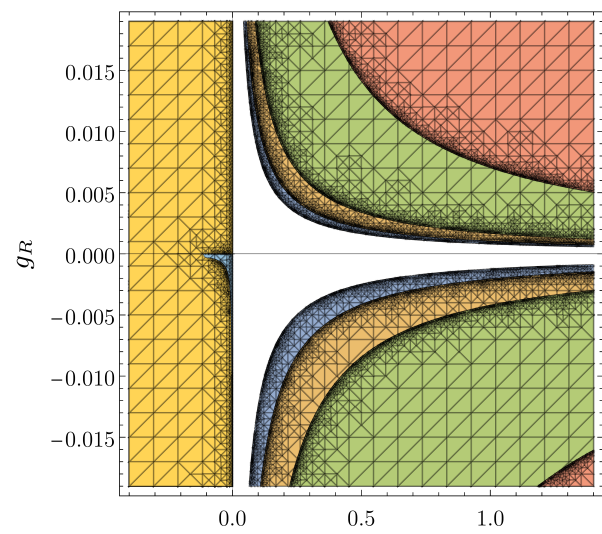

$G \Lambda$

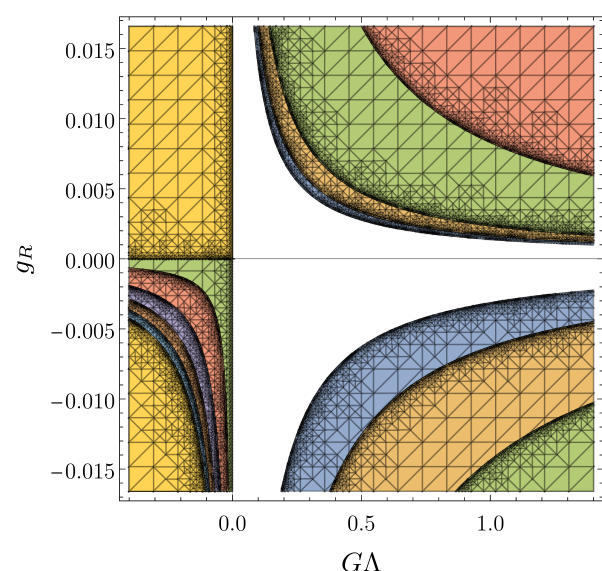

$G \Lambda$

Figure 6. dSC/TCC constraints for $f=0.1$ (top panel), $f=0.5$ (central panel) and $f=1$ (bottom panel), and various values of $c$. Due to the inverse relation in Equation (28) between the inflaton mass in Planck units $m^{2} / M_{\mathrm{Pl}}^{2}$ and the coupling $g_{R}$, the linear bounds in the $\left(m^{2}, \Lambda\right)$ plane translate into hyperbolas in the $\left(g_{R}, G \Lambda\right)$ plane. 

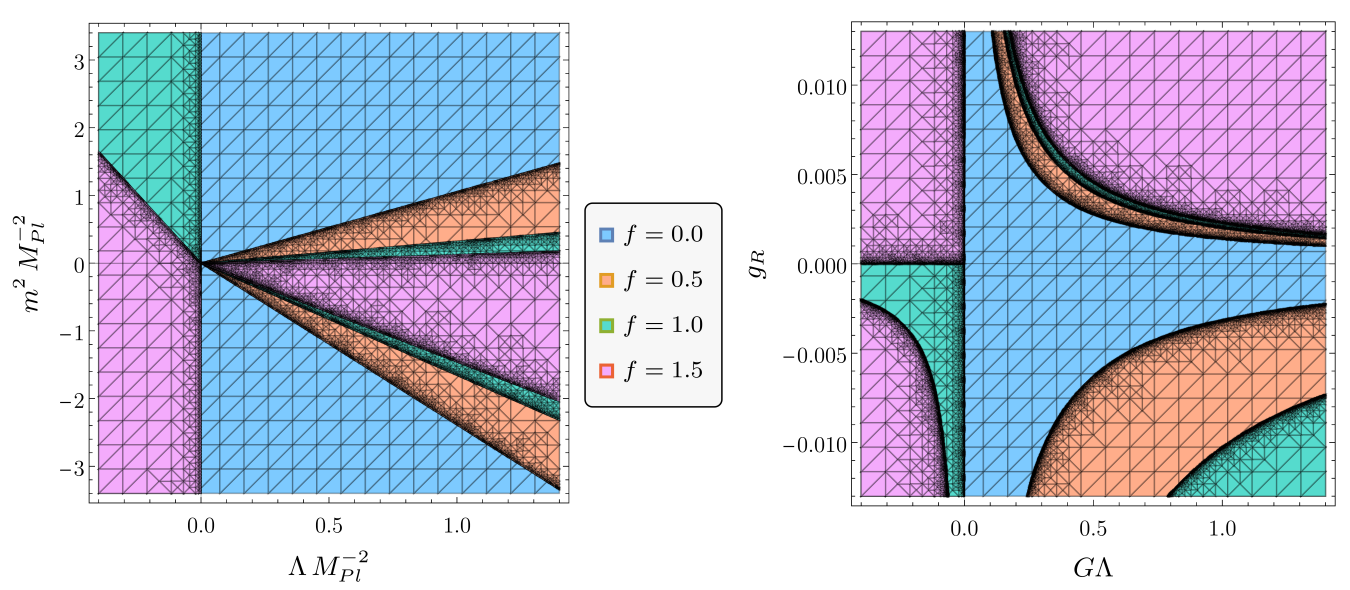

Figure 7. TCC constraints, corresponding to $c=\sqrt{2 / 3}$, for various values of $f$. The bounds are not qualitatively different from the dSC bounds displayed in Figure 6.

\subsection{Intersections of Allowed Regions: Compatibility of Asymptotic Safety with dS, TC and WG Conjectures}

We are now ready to collect the results that we have discussed in the preceding sections, and to visualize the intersection between the different allowed regions. Within (an extrapolation of the) one-loop approximation, asymptotic safety of the RG flow constrains the physical IR parameters of Equation (22) to lie on the plane of Equation (23). On the other hand, while the (duality-invariant) WGC does not entail any additional constraint, the dSC/TCC conditions for the inflaton potential place constraints on the cosmological constant and the inflaton mass. One can plot the intersections for any values of the $\mathcal{O}(1)$ constants $c$ and $f$, and Figure 8, the main result of our work, refers to the representative choice $c=f=1$. Albeit difficult to visualize, there is in general a region of the asymptotically safe plane that appears compatible with the swampland constraints that we have investigated. One can straightforwardly verify that the same conclusion is reached for different values of $f$ and $c$. Our findings, based on the quadratic one-loop approximation, thus point at a non-trivial compatibility between the conditions for UV-completeness dictated by asymptotic safety and some of the most relevant swampland conjectures. Consequently, it also points at the possibility, partially supported by [200,201], of a connection between the frameworks of asymptotic safety and string theory [81]. Within this picture, field-theoretical asymptotic safety would serve as a "pivot" for the RG flow from string theory to low-energy gravity, in the sense that below a certain scale the flow of string theory toward the IR closely approaches a field-theoretical trajectory controlled by a UV fixed point. 



Figure 8. Intersections between the regions allowed by asymptotic safety, the WGC and the dSC/TCC for the representative values $c=f=1$. The WGC bound corresponds to the yellow region, while the $\mathrm{dSC} / \mathrm{TCC}$ bound corresponds to the blue region. The green region depicts the space of IR parameters spanned by asymptotically safe trajectories, which lies within the region allowed by the WGC.

\section{Conclusions}

In this paper we have analyzed the intersection of consistency conditions for Wilson coefficients of gravitational EFTs, combining the constraints of asymptotic safety and swampland conjectures. In particular, in Section 3 we have employed a systematic method to extract the hypersurface of allowed IR parameters stemming from UV-complete RG trajectories in gravitational theories by randomly sampling its relevant deformations, and we have applied this technique to the flow equations stemming from one-loop quadratic gravity [178]. Despite expecting that this approximation be reliable in the IR, the resulting RG flow exhibits a UV non-Gaussian fixed point, consistently with more refined functional RG computations [202-204]. However, the dimension of its critical surface is larger than what is suggested by the functional RG $[66,89,192,205]$. As we have discussed in detail in Section 4, our findings suggest that the requirement that the RG flow be asymptotically safe constrains the physical parameters to lie on a plane, which we have determined, within our one-loop framework, to a precision of order $\mathcal{O}\left(10^{-8}\right)$. The values of the cosmological constant in Planck units seems not to be restricted by these considerations, while the classically marginal couplings lie on a line.

In Sections 4.2 and 4.3 we have investigated the constraints on the Wilson coefficients arising from the weak gravity conjecture (WGC), the de Sitter conjecture (dSC) and the transPlanckian censorship conjecture (TCC). In particular, the WGC does not entail additional 
bounds and is compatible with the UV-complete RG trajectories, while the dSC/TCC bounds are more restrictive. To wit, the Starobinsky-like scalar potential stemming from the (local sector of the) effective action involves the ratio of the cosmological constant to the (squared) inflaton mass, and therefore the corresponding bounds place constraints on the dimensionless ratios $\Lambda / M_{\mathrm{Pl}}^{2}$ and $m / M_{\mathrm{Pl}}$. These bounds depend on some dimensionless $\mathcal{O}(1)$ constants, which we have varied to some extent in our analysis, and generally trace out a region in the plane allowed by asymptotic safety. While we expect that the qualitative results be unaffected by improving the truncation scheme, at least to some extent, it would be interesting to investigate the quantitative deviations in this respect.

While in this work we have focused on the local sector of the effective action, our computation also yields the coefficients of non-local logarithmic form factors, akin to those arising from non-local heat kernel computations [206-211]. It would be interesting to explore their consequences and their role within asymptotically safe gravity [182-184] and their connection to massive matter fields [212].

All in all, our results suggest that swampland constraints can be compatible with restrictions coming from UV completeness of the RG flow, but in a non-trivial fashion: the allowed parameter space is restricted to a non-trivial intersection. In retrospect, one could have expected this result on the grounds that some swampland criteria purport to be necessary conditions for UV completeness that cannot be derived from purely fieldtheoretical considerations, and thus they could constrain further the parameter space compatible with asymptotic safety. On the other hand, the one-loop approximation that we have studied already features the appearance of non-local form factors. In general, non-locality at the level of the effective action is a feature of any standard (local) QFT, and thus it is in principle unrelated to possible fundamental non-localities in the bare (fixedpoint) action. Precisely how the notion of (non-)locality is realized in quantum gravity is an open and intriguing question, partly related to the problem of observables [213-217]. However, a number of semi-classical considerations [162,218-223] point to the breaking of the familiar concept of locality microscopically. Whether asymptotically safe gravity is realized by a bare action polynomial in derivatives (and thus "local" in some sense) is not established yet. Should fundamental non-locality turn out to emerge as a feature of asymptotic safety, this would strengthen its potential connections with the frameworks of non-local gravity [224-227] and string theory [228].

Due to the nature of our approximations, this work constitutes only a first step toward determining whether the asymptotic safety scenario is compatible with the peculiar behavior and UV/IR mixing that gravity could exhibit already at the semi-classical level due to black holes (see [229] for a very recent discussion on their validity and limitations), or with general indications from string theory. In particular, a possible connection between asymptotically safe gravity and string theory has been conjectured in [81], and it is tempting to speculate that it could explain our findings. Computations combining the functional renormalization group techniques [83] with symmetries of string theory [230-235] have provided preliminary evidence in favour of this scenario [200,201]. This possibility extends to the more general notion of "effective asymptotic safety" [236], and swampland bounds could further constrain which RG flows controlled by the "effective" fixed point are closely approached by the RG flow arising from the proper UV completion in the IR.

Most prominently, the absence of continuous global symmetries ${ }^{12}$ is supported by a variety of arguments from black-hole physics, string theory and holography [60-63,239-241], and it would be interesting to explore this foundational issue further in the direction that we have outlined in this paper.

Author Contributions: All authors contributed equally to this article. All authors have read and agreed to the published version of the manuscript.

Funding: The work of I.B. is supported by the Fonds de la Recherche Scientifique-FNRS under Grants No. F.4503.20 ("HighSpinSymm") and T.0022.19 ("Fundamental issues in extended gravitational theories"). A.P. acknowledges support by Perimeter Institute for Theoretical Physics. Research 
at Perimeter Institute is supported in part by the Government of Canada through the Department of Innovation, Science and Economic Development and by the Province of Ontario through the Ministry of Colleges and Universities.

Institutional Review Board Statement: Not applicable.

Informed Consent Statement: Not applicable.

Data Availability Statement: Not applicable.

Acknowledgments: The authors would like to thank F. Saueressig for insighful discussions and B. Knorr for feedback on the manuscript. The authors thank also B. Holdom for spotting a typo.

Conflicts of Interest: The authors declare no conflict of interest.

\section{Notes}

See also [81] for related discussions on the weak gravity conjecture in the context of asymptotically safe gravity.

Nevertheless, scenarios with high-energy supersymmetry breaking have been investigated in the context of the swampland $[24,25,146]$. See [147-149] for recent reviews.

See $[150,151]$ for reviews.

For Einstein-Maxwell theory, the extremality bound reads $\frac{M}{Q} \geq \sqrt{2} M_{\mathrm{Pl}}$.

Another instance of the interplay between duality and the WGC has been studied in [170].

See also [172-175] for discussions on eternal inflation and the swampland.

Let us remark that here "quadratic" refers to the order in the curvatures. In terms of derivatives, the action in Equation (11) is quartic.

8 Note that, since we are interested in the IR regime, our convention for the RG time is such that $t \rightarrow+\infty$ in the IR.

9 Although 2 and 4 are not the canonical mass dimensions of $G$ and $\Lambda$, they are the canonical dimension of the couplings $1 / G$ and $\Lambda / G$ that multiply the operators $\sqrt{-g}$ and $\sqrt{-g} R$. This occurs because the transformation between these couplings is non-singular, as explained in [180]. On the other hand, at the Gaussian fixed point the transformation between the couplings is singular, and the dimensions change accordingly.

10 Notice that our convention for the Planck mass differs from the more widespread "reduced" Planck mass $\widehat{M}_{\mathrm{Pl}}^{-2}=8 \pi G$.

11 One exception could be a non-local form factor of the type $\sim 1 / \square$, as discussed in $[67,68]$.

12 The fate of global discrete symmetries has been investigated in the context of asymptotically safe gravity in $[237,238]$.

\section{References}

1. 't Hooft, G.; Veltman, M.J.G. One loop divergencies in the theory of gravitation. Ann. Inst. Henri Poincare Phys. Theor. A 1974, 20, 69-94.

2. Goroff, M.H.; Sagnotti, A. The ultraviolet behavior of Einstein gravity. Nucl. Phys. B 1986, 266, 709-736. [CrossRef]

3. Goroff, M.H.; Sagnotti, A. Quantum gravity at two loops. Phys. Lett. B 1985, 160, 81-86. [CrossRef]

4. Vafa, C. The String landscape and the swampland. arXiv 2005, arXiv:hep-th/0509212.

5. Berglund, P.; Mayr, P. Non-perturbative superpotentials in F-theory and string duality. J. High Energy Phys. 2013, 1, 114. [CrossRef]

6. Gonzalo, E.; Ibáñez, L.E.; Uranga, A.M. Modular symmetries and the swampland conjectures. J. High Energy Phys. $2019,5,105$. [CrossRef]

7. Marchesano, F.; Wiesner, M. Instantons and infinite distances. J. High Energy Phys. 2019, 8, 088. [CrossRef]

8. Blumenhagen, R.; Brinkmann, M.; Makridou, A. Quantum Log-Corrections to Swampland Conjectures. J. High Energy Phys. 2020, 2, 064. [CrossRef]

9. Baume, F.; Marchesano, F.; Wiesner, M. Instanton Corrections and Emergent Strings. J. High Energy Phys. 2020, 4, 174. [CrossRef]

10. Palti, E.; Vafa, C.; Weigand, T. Supersymmetric Protection and the Swampland. J. High Energy Phys. 2020, 6, 168. [CrossRef]

11. Marchesano, F.; Prieto, D.; Wiesner, M. F-theory flux vacua at large complex structure. arXiv 2021, arXiv:2105.09326.

12. Lee, S.J.; Lerche, W.; Weigand, T. Tensionless Strings and the Weak Gravity Conjecture. J. High Energy Phys. 2018, 10, 164. [CrossRef]

13. Lee, S.J.; Lerche, W.; Weigand, T. A Stringy Test of the Scalar Weak Gravity Conjecture. Nucl. Phys. B 2019, 938, 321-350. [CrossRef]

14. Lee, S.J.; Lerche, W.; Weigand, T. Modular Fluxes, Elliptic Genera, and Weak Gravity Conjectures in Four Dimensions. J. High Energy Phys. 2019, 8, 104. [CrossRef] 
15. Lee, S.J.; Lerche, W.; Weigand, T. Emergent Strings, Duality and Weak Coupling Limits for Two-Form Fields. arXiv 2019, arXiv:1904.06344.

16. Klaewer, D.; Lee, S.J.; Weigand, T.; Wiesner, M. Quantum corrections in $4 \mathrm{~d} N=1$ infinite distance limits and the weak gravity conjecture. J. High Energy Phys. 2021, 3, 252. [CrossRef]

17. Klaewer, D. Modular Curves and the Refined Distance Conjecture. arXiv 2021, arXiv:2108.00021.

18. Grimm, T.W.; Palti, E.; Valenzuela, I. Infinite Distances in Field Space and Massless Towers of States. J. High Energy Phys. 2018, 8, 143. [CrossRef]

19. Grimm, T.W.; Van De Heisteeg, D. Infinite Distances and the Axion Weak Gravity Conjecture. J. High Energy Phys. 2020, 3, 020. [CrossRef]

20. Grimm, T.W.; Li, C.; Valenzuela, I. Asymptotic Flux Compactifications and the Swampland. J. High Energy Phys. 2020, 6, 009; Erratum in 2021, 1, 007. [CrossRef]

21. Gendler, N.; Valenzuela, I. Merging the weak gravity and distance conjectures using BPS extremal black holes. J. High Energy Phys. 2021, 1, 176. [CrossRef]

22. Grimm, T.W.; Li, C. Universal axion backreaction in flux compactifications. J. High Energy Phys. 2021, 6, 067. [CrossRef]

23. Bastian, B.; Grimm, T.W.; van de Heisteeg, D. Modelling General Asymptotic Calabi-Yau Periods. arXiv 2021, arXiv:2105.02232.

24. Basile, I.; Lanza, S. de Sitter in non-supersymmetric string theories: No-go theorems and brane-worlds. J. High Energy Phys. 2020, 10, 108. [CrossRef]

25. Basile, I. Supersymmetry breaking, brane dynamics and Swampland conjectures. arXiv 2021, arXiv:2106.04574.

26. Kachru, S.; Kallosh, R.; Linde, A.D.; Trivedi, S.P. De Sitter vacua in string theory. Phys. Rev. D 2003, 68, 046005. [CrossRef]

27. Balasubramanian, V.; Berglund, P.; Conlon, J.P.; Quevedo, F. Systematics of moduli stabilisation in Calabi-Yau flux compactifications. J. High Energy Phys. 2005, 3, 007. [CrossRef]

28. Koerber, P.; Martucci, L. From ten to four and back again: How to generalize the geometry. J. High Energy Phys. 2007, 8, 059. [CrossRef]

29. Danielsson, U.H.; Haque, S.S.; Shiu, G.; Van Riet, T. Towards Classical de Sitter Solutions in String Theory. J. High Energy Phys. 2009, 9, 114. [CrossRef]

30. Moritz, J.; Retolaza, A.; Westphal, A. Toward de Sitter space from ten dimensions. Phys. Rev. D 2018, 97, 046010. [CrossRef]

31. Kallosh, R.; Wrase, T. dS Supergravity from 10d. Fortsch. Phys. 2019, 67, 1800071. [CrossRef]

32. Bena, I.; Dudas, E.; Graña, M.; Lüst, S. Uplifting Runaways. Fortsch. Phys. 2019, 67, 1800100. [CrossRef]

33. Gautason, F.; Van Hemelryck, V.; Van Riet, T. The Tension between 10D Supergravity and dS Uplifts. Fortsch. Phys. 2019, 67, 1800091. [CrossRef]

34. Córdova, C.; De Luca, G.B.; Tomasiello, A. Classical de Sitter Solutions of 10-Dimensional Supergravity. Phys. Rev. Lett. 2019, 122, 091601. [CrossRef] [PubMed]

35. Blåbäck, J.; Danielsson, U.; Dibitetto, G.; Giri, S. Constructing stable de Sitter in M-theory from higher curvature corrections. J. High Energy Phys. 2019, 9, 042. [CrossRef]

36. Hamada, Y.; Hebecker, A.; Shiu, G.; Soler, P. Understanding KKLT from a 10d perspective. J. High Energy Phys. 2019, 6, 019. [CrossRef]

37. Gautason, F.; Van Hemelryck, V.; Van Riet, T.; Venken, G. A 10d view on the KKLT AdS vacuum and uplifting. J. High Energy Phys. 2020, 6, 074. [CrossRef]

38. Cribiori, N.; Junghans, D. No classical (anti-)de Sitter solutions with O8-planes. Phys. Lett. B 2019, 793, 54-58. [CrossRef]

39. Andriot, D. Open problems on classical de Sitter solutions. Fortsch. Phys. 2019, 67, 1900026. [CrossRef]

40. Shukla, P. T-dualizing de Sitter no-go scenarios. Phys. Rev. D 2020, 102, 026014. [CrossRef]

41. Shukla, P. Rigid nongeometric orientifolds and the swampland. Phys. Rev. D 2021, 103, 086010. [CrossRef]

42. Córdova, C.; De Luca, G.B.; Tomasiello, A. New de Sitter Solutions in Ten Dimensions and Orientifold Singularities. arXiv 2019, arXiv:1911.04498.

43. Andriot, D.; Marconnet, P.; Wrase, T. New de Sitter solutions of 10d type IIB supergravity. J. High Energy Phys. 2020, 8, 076. [CrossRef]

44. Andriot, D.; Marconnet, P.; Wrase, T. Intricacies of classical de Sitter string backgrounds. arXiv 2020, arXiv:2006.01848.

45. Farakos, F.; Kehagias, A.; Liatsos, N. de Sitter decay through goldstino evaporation. J. High Energy Phys. 2021, 2, 186. [CrossRef]

46. Gao, X.; Hebecker, A.; Junghans, D. Control issues of KKLT. Fortsch. Phys. 2020, 68, 2000089. [CrossRef]

47. Bena, I.; De Luca, G.B.; Graña, M.; Lo Monaco, G. Oh, wait, O8 de Sitter may be unstable! J. High Energy Phys. 2021, 3, 168. [CrossRef]

48. Bena, I.; Blåbäck, J.; Graña, M.; Lüst, S. The Tadpole Problem. arXiv 2020, arXiv:2010.10519.

49. Crinò, C.; Quevedo, F.; Valandro, R. On de Sitter String Vacua from Anti-D3-Branes in the Large Volume Scenario. J. High Energy Phys. 2021, 3, 258. [CrossRef]

50. Dine, M.; Law-Smith, J.A.P.; Sun, S.; Wood, D.; Yu, Y. Obstacles to Constructing de Sitter Space in String Theory. J. High Energy Phys. 2021, 2, 050. [CrossRef]

51. Basiouris, V.; Leontaris, G.K. Note on de Sitter vacua from perturbative and non-perturbative dynamics in type IIB/F-theory compactifications. Phys. Lett. B 2020, 810, 135809. [CrossRef]

52. Cribiori, N.; Dall'agata, G.; Farakos, F. Weak gravity versus de Sitter. J. High Energy Phys. 2021, 4, 046. [CrossRef] 
53. Hebecker, A.; Leonhardt, S. Winding Uplifts and the Challenges of Weak and Strong SUSY Breaking in AdS. J. High Energy Phys. 2021, 3, 284. [CrossRef]

54. Andriot, D. Tachyonic de Sitter solutions of 10d type II supergravities. arXiv 2021, arXiv:2101.06251.

55. De Luca, G.B.; Silverstein, E.; Torroba, G. Hyperbolic compactification of M-theory and de Sitter quantum gravity. arXiv 2021, arXiv:2104.13380.

56. Cicoli, M.; Etxebarria, I.n.G.; Quevedo, F.; Schachner, A.; Shukla, P.; Valandro, R. The Standard Model Quiver in de Sitter String Compactifications. arXiv 2021, arXiv:2106.11964.

57. Cribiori, N.; Junghans, D.; Van Hemelryck, V.; Van Riet, T.; Wrase, T. Scale-separated AdS 4 vacua of IIA orientifolds and M-theory. arXiv 2021, arXiv:2107.00019.

58. Dine, M.; Seiberg, N. Is the Superstring Weakly Coupled? Phys. Lett. B 1985, 162, 299-302. [CrossRef]

59. Montero, M.; Vafa, C. Cobordism Conjecture, Anomalies, and the String Lamppost Principle. J. High Energy Phys. 2021, 1, 063. [CrossRef]

60. Misner, C.W.; Wheeler, J.A. Classical physics as geometry: Gravitation, electromagnetism, unquantized charge, and mass as properties of curved empty space. Ann. Phys. 1957, 2, 525-603. [CrossRef]

61. Polchinski, J. Monopoles, duality, and string theory. Int. J. Mod. Phys. A 2004, 19S1, 145-156. [CrossRef]

62. Banks, T.; Seiberg, N. Symmetries and Strings in Field Theory and Gravity. Phys. Rev. D 2011, 83, 084019. [CrossRef]

63. Harlow, D.; Ooguri, H. Symmetries in quantum field theory and quantum gravity. Commun. Math. Phys. 2021, 383, 1669-1804. [CrossRef]

64. Arkani-Hamed, N.; Motl, L.; Nicolis, A.; Vafa, C. The String landscape, black holes and gravity as the weakest force. J. High Energy Phys. 2007, 6, 060. [CrossRef]

65. Benedetti, D.; Groh, K.; Machado, P.F.; Saueressig, F. The universal RG machine. J. High Energy Phys. 2011, 6, 79. [CrossRef]

66. Knorr, B. The derivative expansion in asymptotically safe quantum gravity: General setup and quartic order. arXiv 2021, arXiv:2104.11336

67. Belgacem, E.; Dirian, Y.; Foffa, S.; Maggiore, M. Nonlocal gravity. Conceptual aspects and cosmological predictions. J. Cosmol. Astropart. Phys. 2018, 3, 002. [CrossRef]

68. Knorr, B.; Saueressig, F. Towards reconstructing the quantum effective action of gravity. Phys. Rev. Lett. 2018, 121, 161304. [CrossRef]

69. Guerrieri, A.; Penedones, J.; Vieira, P. Where is String Theory? arXiv 2021, arXiv:2102.02847.

70. Caron-Huot, S.; Mazac, D.; Rastelli, L.; Simmons-Duffin, D. Sharp Boundaries for the Swampland. arXiv 2021, arXiv:2102.08951.

71. de Rham, C.; Melville, S.; Tolley, A.J.; Zhou, S.Y. UV complete me: Positivity Bounds for Particles with Spin. J. High Energy Phys. 2018, 3, 011. [CrossRef]

72. de Rham, C.; Melville, S.; Tolley, A.J. Improved Positivity Bounds and Massive Gravity. J. High Energy Phys. 2018,4 , 083. [CrossRef]

73. de Rham, C.; Melville, S.; Tolley, A.J.; Zhou, S.Y. Positivity Bounds for Massive Spin-1 and Spin-2 Fields. J. High Energy Phys. 2019, 3, 182. [CrossRef]

74. De Rham, C.; Heisenberg, L.; Tolley, A.J. Spin-2 fields and the weak gravity conjecture. Phys. Rev. D 2019, 100, 104033. [CrossRef]

75. Alberte, L.; de Rham, C.; Momeni, A.; Rumbutis, J.; Tolley, A.J. EFT of Interacting Spin-2 Fields. J. High Energy Phys. $2020,1,131$. [CrossRef]

76. Alberte, L.; de Rham, C.; Momeni, A.; Rumbutis, J.; Tolley, A.J. Positivity Constraints on Interacting Spin-2 Fields. J. High Energy Phys. 2020, 3, 097. [CrossRef]

77. Alberte, L.; de Rham, C.; Momeni, A.; Rumbutis, J.; Tolley, A.J. Positivity Constraints on Interacting Pseudo-Linear Spin-2 Fields. J. High Energy Phys. 2020, 7, 121. [CrossRef]

78. Alberte, L.; de Rham, C.; Jaitly, S.; Tolley, A.J. QED positivity bounds. Phys. Rev. D 2021, 103, 125020. [CrossRef]

79. Alberte, L.; de Rham, C.; Jaitly, S.; Tolley, A.J. Positivity Bounds and the Massless Spin-2 Pole. Phys. Rev. D 2020, 102, 125023. [CrossRef]

80. de Rham, C.; Melville, S.; Noller, J. Positivity Bounds on Dark Energy: When Matter Matters. arXiv 2021, arXiv:2103.06855.

81. de Alwis, S.; Eichhorn, A.; Held, A.; Pawlowski, J.M.; Schiffer, M.; Versteegen, F. Asymptotic safety, string theory and the weak gravity conjecture. Phys. Lett. B 2019, 798, 134991. [CrossRef]

82. Weinberg, S. Critical Phenomena for Field Theorists. In Erice Subnuclear Physics; Springer: Boston, MA, USA, 1976.

83. Dupuis, N.; Canet, L.; Eichhorn, A.; Metzner, W.; Pawlowski, J.; Tissier, M.; Wschebor, N. The nonperturbative functional renormalization group and its applications. arXiv 2020, arXiv:2006.04853.

84. Souma, W. Non-Trivial Ultraviolet Fixed Point in Quantum Gravity. Prog. Theor. Phys. 1999, 102, 181-195. [CrossRef]

85. Lauscher, O.; Reuter, M. Flow equation of quantum Einstein gravity in a higher-derivative truncation. Phys. Rev. D 2002, 66, 025026. [CrossRef]

86. Litim, D.F. Fixed Points of Quantum Gravity. Phys. Rev. Lett. 2004, 92, 201301. [CrossRef] [PubMed]

87. Codello, A.; Percacci, R. Fixed Points of Higher-Derivative Gravity. Phys. Rev. Lett. 2006, 97, 221301. [CrossRef] [PubMed]

88. Machado, P.F.; Saueressig, F. On the renormalization group flow of f(R)-gravity. Phys. Rev. D 2008, 77, 124045. [CrossRef]

89. Benedetti, D.; Machado, P.F.; Saueressig, F. Asymptotic Safety in Higher-Derivative Gravity. Mod. Phys. Lett. A 2009, 24, 2233-2241. [CrossRef] 
90. Dietz, J.A.; Morris, T.R. Asymptotic safety in the $\mathrm{f}(\mathrm{R})$ approximation. J. High Energy Phys. 2013, 1, 108. [CrossRef]

91. Donà, P.; Eichhorn, A.; Percacci, R. Matter matters in asymptotically safe quantum gravity. Phys. Rev. D 2014, $89,084035$. [CrossRef]

92. Eichhorn, A. On unimodular quantum gravity. Class. Quantum Gravity 2013, 30, 115016. [CrossRef]

93. Donà, P.; Eichhorn, A.; Percacci, R. Consistency of matter models with asymptotically safe quantum gravity. Can. J. Phys. 2015, 93, 988-994. [CrossRef]

94. Christiansen, N.; Knorr, B.; Pawlowski, J.M.; Rodigast, A. Global Flows in Quantum Gravity. Phys. Rev. 2016, D93, 044036. [CrossRef]

95. Falls, K.; Litim, D.; Nikolakopoulos, K.; Rahmede, C. Further evidence for asymptotic safety of quantum gravity. Phys. Rev. D 2016, 93, 104022. [CrossRef]

96. Christiansen, N.; Knorr, B.; Meibohm, J.; Pawlowski, J.M.; Reichert, M. Local Quantum Gravity. Phys. Rev. 2015, D92, 121501. [CrossRef]

97. Meibohm, J.; Pawlowski, J.M.; Reichert, M. Asymptotic safety of gravity-matter systems. Phys. Rev. D 2016, 93, 084035. [CrossRef]

98. Oda, K.Y.; Yamada, M. Non-minimal coupling in Higgs-Yukawa model with asymptotically safe gravity. Class. Quantum Gravity 2016, 33, 125011. [CrossRef]

99. Donà, P.; Eichhorn, A.; Labus, P.; Percacci, R. Asymptotic safety in an interacting system of gravity and scalar matter. Phys. Rev. D 2016, 93, 044049. [CrossRef]

100. Biemans, J.; Platania, A.; Saueressig, F. Quantum gravity on foliated spacetimes: Asymptotically safe and sound. Phys. Rev. 2017, D95, 086013. [CrossRef]

101. Eichhorn, A.; Held, A.; Pawlowski, J.M. Quantum-gravity effects on a Higgs-Yukawa model. Phys. Rev. D 2016, 94, 104027. [CrossRef]

102. Dietz, J.A.; Morris, T.R.; Slade, Z.H. Fixed point structure of the conformal factor field in quantum gravity. Phys. Rev. D 2016, 94, 124014. [CrossRef]

103. Falls, K.; Ohta, N. Renormalization group equation for $\mathrm{f}(\mathrm{R})$ gravity on hyperbolic spaces. Phys. Rev. D 2016, 94, 084005. [CrossRef]

104. Gies, H.; Knorr, B.; Lippoldt, S.; Saueressig, F. Gravitational Two-Loop Counterterm Is Asymptotically Safe. Phys. Rev. Lett. 2016, 116, 211302. [CrossRef]

105. Biemans, J.; Platania, A.; Saueressig, F. Renormalization group fixed points of foliated gravity-matter systems. J. High Energy Phys. 2017, 5, 093. [CrossRef]

106. Christiansen, N.; Litim, D.F.; Pawlowski, J.M.; Reichert, M. Asymptotic safety of gravity with matter. Phys. Rev. 2018, D97, 106012. [CrossRef]

107. Hamada, Y.; Yamada, M. Asymptotic safety of higher derivative quantum gravity non-minimally coupled with a matter system. J. High Energy Phys. 2017, 8, 070. [CrossRef]

108. Platania, A.; Saueressig, F. Functional Renormalization Group Flows on Friedman-Lemaître-Robertson-Walker backgrounds. Found. Phys. 2018, 48, 1291-1304. [CrossRef]

109. Falls, K.; King, C.R.; Litim, D.F.; Nikolakopoulos, K.; Rahmede, C. Asymptotic safety of quantum gravity beyond Ricci scalars. Phys. Rev. 2018, D97, 086006. [CrossRef]

110. De Brito, G.P.; Ohta, N.; Pereira, A.D.; Tomaz, A.A.; Yamada, M. Asymptotic safety and field parametrization dependence in the $f(R)$ truncation. arXiv 2018, arXiv:1805.09656.

111. Eichhorn, A.; Lippoldt, S.; Schiffer, M. Zooming in on fermions and quantum gravity. Phys. Rev. 2019, D99, 086002. [CrossRef]

112. Eichhorn, A.; Schiffer, M. $d=4$ as the critical dimensionality of asymptotically safe interactions. Phys. Lett. 2019, B793, 383-389. [CrossRef]

113. De Brito, G.P.; Eichhorn, A.; Pereira, A.D. A link that matters: Towards phenomenological tests of unimodular asymptotic safety. J. High Energy Phys. 2019, 9, 100. [CrossRef]

114. Donoghue, J.F. A Critique of the Asymptotic Safety Program. Front. Phys. 2020, 8, 56. [CrossRef]

115. Bonanno, A.; Eichhorn, A.; Gies, H.; Pawlowski, J.M.; Percacci, R.; Reuter, M.; Saueressig, F.; Vacca, G.P. Critical reflections on asymptotically safe gravity. Front. Phys. 2020, 8, 269. [CrossRef]

116. Bonanno, A.; Saueressig, F. Asymptotically safe cosmology-A status report. C. R. Phys. 2017, 18, 254-264. [CrossRef]

117. Platania, A. From renormalization group flows to cosmology. Front. Phys. 2020, 8, 188. [CrossRef]

118. Bonanno, A.; Reuter, M. Spacetime structure of an evaporating black hole in quantum gravity. Phys. Rev. 2006, D73, 083005 . [CrossRef]

119. Falls, K.; Litim, D.F. Black hole thermodynamics under the microscope. Phys. Rev. 2014, D89, 084002. [CrossRef]

120. Torres, R.; Fayos, F. On the quantum corrected gravitational collapse. Phys. Lett. B 2015, 747, 245-250. [CrossRef]

121. Koch, B.; Rioseco, P. Black Hole Solutions for Scale Dependent Couplings: The de Sitter and the Reissner-Nordström Case. Class. Quantum Gravity 2016, 33, 035002. [CrossRef]

122. Bonanno, A.; Platania, A. Asymptotically safe inflation from quadratic gravity. Phys. Lett. 2015, B750, 638-642. [CrossRef]

123. Bonanno, A.; Platania, A. Asymptotically Safe R+R ${ }^{2}$ gravity. PoS 2016, corfu2015, 159. [CrossRef]

124. Kofinas, G.; Zarikas, V. Asymptotically Safe gravity and non-singular inflationary Big Bang with vacuum birth. Phys. Rev. 2016, D94, 103514. [CrossRef] 
125. Falls, K.; Litim, D.F.; Nikolakopoulos, K.; Rahmede, C. On de Sitter solutions in asymptotically safe $f(R)$ theories. Class. Quantum Gravity 2018, 35, 135006. [CrossRef]

126. Bonanno, A.; Koch, B.; Platania, A. Cosmic Censorship in Quantum Einstein Gravity. Class. Quantum Gravity 2017, $34,095012$. [CrossRef]

127. Bonanno, A.; Gabriele Gionti, S.J.; Platania, A. Bouncing and emergent cosmologies from Arnowitt-Deser-Misner RG flows. Class. Quantum Gravity 2018, 35, 065004. [CrossRef]

128. Bonanno, A.; Koch, B.; Platania, A. Asymptotically Safe gravitational collapse: Kuroda-Papapetrou RG-improved model. PoS 2017, corfu2016, 058. [CrossRef]

129. Bonanno, A.; Koch, B.; Platania, A. Gravitational collapse in Quantum Einstein Gravity. arXiv 2017, arXiv:1710.10845. [CrossRef]

130. Bonanno, A.; Platania, A.; Saueressig, F. Cosmological bounds on the field content of asymptotically safe gravity-matter models. arXiv 2018, arXiv:1803.02355.

131. Liu, L.H.; Prokopec, T.; Starobinsky, A.A. Inflation in an effective gravitational model and asymptotic safety. Phys. Rev. 2018, D98, 043505. [CrossRef]

132. Majhi, A. Singularity from star collapse, torsion and asymptotic safety of gravity. arXiv 2018, arXiv:1804.00960.

133. Anagnostopoulos, F.K.; Basilakos, S.; Kofinas, G.; Zarikas, V. Constraining the Asymptotically Safe Cosmology: Cosmic acceleration without dark energy. J. Cosmol. Astropart. Phys. 2019, 1902, 053. [CrossRef]

134. Adeifeoba, A.; Eichhorn, A.; Platania, A. Towards conditions for black-hole singularity-resolution in asymptotically safe quantum gravity. Class. Quantum Gravity 2018, 35, 225007. [CrossRef]

135. Pawlowski, J.M.; Stock, D. Quantum-improved Schwarzschild-(A)dS and Kerr-(A)dS spacetimes. Phys. Rev. 2018, D98, 106008. [CrossRef]

136. Gubitosi, G.; Ooijer, R.; Ripken, C.; Saueressig, F. Consistent early and late time cosmology from the RG flow of gravity. J. Cosmol. Astropart. Phys. 2018, 1812, 004. [CrossRef]

137. Platania, A. The inflationary mechanism in Asymptotically Safe Gravity. Universe 2019, 5, 189. [CrossRef]

138. Platania, A. Dynamical renormalization of black-hole spacetimes. Eur. Phys. J. 2019, C79, 470. [CrossRef]

139. Bonanno, A.; Casadio, R.; Platania, A. Gravitational antiscreening in stellar interiors. J. Cosmol. Astropart. Phys. 2020, $2001,022$. [CrossRef]

140. Held, A.; Gold, R.; Eichhorn, A. Asymptotic safety casts its shadow. J. Cosmol. Astropart. Phys. 2019, 1906, 029. [CrossRef]

141. Bosma, L.; Knorr, B.; Saueressig, F. Resolving Spacetime Singularities within Asymptotic Safety. Phys. Rev. Lett. 2019, $123,101301$. [CrossRef]

142. Niedermaier, M.R. Gravitational Fixed Points from Perturbation Theory. Phys. Rev. Lett. 2009, 103, 101303. [CrossRef]

143. Niedermaier, M. Gravitational fixed points and asymptotic safety from perturbation theory. Nucl. Phys. B 2010, 833, 226-270. [CrossRef]

144. Cribiori, N.; Lust, D.; Scalisi, M. The gravitino and the swampland. J. High Energy Phys. 2021, 6, 071. [CrossRef]

145. Castellano, A.; Font, A.; Herraez, A.; Ibáñez, L.E. A Gravitino Distance Conjecture. arXiv 2021, arXiv:2104.10181.

146. Bonnefoy, Q.; Dudas, E.; Lüst, S. On the weak gravity conjecture in string theory with broken supersymmetry. Nucl. Phys. B 2019, 947, 114738. [CrossRef]

147. Mourad, J.; Sagnotti, A. An Update on Brane Supersymmetry Breaking. arXiv 2017, arXiv:1711.11494.

148. Basile, I. Supersymmetry Breaking and Stability in String Vacua: Brane dynamics, bubbles and the swampland. La Rivista del Nuovo Cimento 2021, 1, 98. [CrossRef]

149. Mourad, J.; Sagnotti, A. String (In)Stability Issues with Broken Supersymmetry. arXiv 2021, arXiv:2107.04064.

150. Palti, E. The Swampland: Introduction and Review. Fortsch. Phys. 2019, 67, 1900037. [CrossRef]

151. van Beest, M.; Calderón-Infante, J.; Mirfendereski, D.; Valenzuela, I. Lectures on the Swampland Program in String Compactifications. arXiv 2021, arXiv:2102.01111.

152. Andriot, D.; Cribiori, N.; Erkinger, D. The web of swampland conjectures and the TCC bound. J. High Energy Phys. 2020, 7, 162. [CrossRef]

153. Lanza, S.; Marchesano, F.; Martucci, L.; Valenzuela, I. Swampland Conjectures for Strings and Membranes. J. High Energy Phys. 2021, 2, 006. [CrossRef]

154. Ooguri, H.; Vafa, C. On the Geometry of the String Landscape and the Swampland. Nucl. Phys. B 2007, 766, 21-33. [CrossRef]

155. Ooguri, H.; Palti, E.; Shiu, G.; Vafa, C. Distance and de Sitter Conjectures on the Swampland. Phys. Lett. B 2019, 788, 180-184. [CrossRef]

156. Graña, M.; Herráez, A. The Swampland Conjectures: A bridge from Quantum Gravity to Particle Physics. arXiv 2021, arXiv:2107.00087.

157. Obied, G.; Ooguri, H.; Spodyneiko, L.; Vafa, C. De Sitter Space and the Swampland. arXiv 2018, arXiv:1806.08362.

158. Garg, S.K.; Krishnan, C. Bounds on Slow Roll and the de Sitter Swampland. J. High Energy Phys. 2019, 11, 075. [CrossRef]

159. Andriot, D.; Roupec, C. Further refining the de Sitter swampland conjecture. Fortsch. Phys. 2019, 67, 1800105. [CrossRef]

160. Bedroya, A.; Vafa, C. Trans-Planckian Censorship and the Swampland. J. High Energy Phys. 2020, 9, 123. [CrossRef]

161. Brandenberger, R. Trans-Planckian Censorship Conjecture and Early Universe Cosmology. arXiv 2021, arXiv:2102.09641.

162. Giddings, S.B. Black holes and massive remnants. Phys. Rev. 1992, D46, 1347-1352. [CrossRef]

163. Susskind, L. Trouble for remnants. arXiv 1995, arXiv:hep-th/9501106. 
164. Kats, Y.; Motl, L.; Padi, M. Higher-order corrections to mass-charge relation of extremal black holes. J. High Energy Phys. 2007, 12, 068. [CrossRef]

165. Cheung, C.; Liu, J.; Remmen, G.N. Proof of the Weak Gravity Conjecture from Black Hole Entropy. J. High Energy Phys. 2018, 10, 004. [CrossRef]

166. Charles, A.M.; Larsen, F.; Mayerson, D.R. Non-Renormalization For Non-Supersymmetric Black Holes. J. High Energy Phys. 2017, 8, 048. [CrossRef]

167. Hamada, Y.; Noumi, T.; Shiu, G. Weak Gravity Conjecture from Unitarity and Causality. Phys. Rev. Lett. 2019, $123,051601$. [CrossRef]

168. Charles, A.M. The Weak Gravity Conjecture, RG Flows, and Supersymmetry. arXiv 2019, arXiv:1906.07734.

169. Cano, P.A.; Murcia, A. Duality-invariant extensions of Einstein-Maxwell theory. arXiv 2021, arXiv:2104.07674.

170. Loges, G.J.; Noumi, T.; Shiu, G. Duality and Supersymmetry Constraints on the Weak Gravity Conjecture. J. High Energy Phys. 2020, 11, 008. [CrossRef]

171. Kinney, W.H.; Vagnozzi, S.; Visinelli, L. The zoo plot meets the swampland: Mutual (in)consistency of single-field inflation, string conjectures, and cosmological data. Class. Quantum Gravity 2019, 36, 117001. [CrossRef]

172. Rudelius, T. Dimensional Reduction and (Anti) de Sitter Bounds. arXiv 2021, arXiv:2101.11617.

173. Rudelius, T. Conditions for (No) Eternal Inflation. J. Cosmol. Astropart. Phys. 2019, 8, 009. [CrossRef]

174. Chojnacki, J.; Krajecka, J.; Kwapisz, J.H.; Slowik, O.; Strag, A. Is asymptotically safe inflation eternal? J. Cosmol. Astropart. Phys. 2021, 4, 076. [CrossRef]

175. Jonas, C.; Lehners, J.L.; Quintin, J. Cosmological consequences of a principle of finite amplitudes. Phys. Rev. D 2021, 103, 103525. [CrossRef]

176. Lüst, D.; Palti, E.; Vafa, C. AdS and the Swampland. Phys. Lett. B 2019, 797, 134867. [CrossRef]

177. Bedroya, A.; Brandenberger, R.; Loverde, M.; Vafa, C. Trans-Planckian Censorship and Inflationary Cosmology. Phys. Rev. D 2020, 101, 103502. [CrossRef]

178. Codello, A.; Percacci, R.; Rahmede, C. Investigating the ultraviolet properties of gravity with a Wilsonian renormalization group equation. Ann. Phys. 2009, 324, 414-469. [CrossRef]

179. Saueressig, F.; Groh, K.; Rechenberger, S.; Zanusso, O. Higher Derivative Gravity from the Universal Renormalization Group Machine. arXiv 2011, arXiv:1111.1743.

180. Percacci, R. Asymptotic Safety. arXiv 2007, arXiv:0709.3851.

181. Litim, D.; Satz, A. Limit cycles and quantum gravity. arXiv 2012, arXiv:1205.4218.

182. Knorr, B.; Ripken, C.; Saueressig, F. Form Factors in Asymptotic Safety: Conceptual ideas and computational toolbox. Class. Quantum Gravity 2019, 36, 234001. [CrossRef]

183. Draper, T.; Knorr, B.; Ripken, C.; Saueressig, F. Finite Quantum Gravity Amplitudes: No Strings Attached. Phys. Rev. Lett. 2020, 125, 181301. [CrossRef]

184. Draper, T.; Knorr, B.; Ripken, C.; Saueressig, F. Graviton-Mediated Scattering Amplitudes from the Quantum Effective Action. J. High Energy Phys. 2020, 11, 136. [CrossRef]

185. Riegert, R.J. A Nonlocal Action for the Trace Anomaly. Phys. Lett. B 1984, 134, 56-60. [CrossRef]

186. Deser, S. Conformal anomalies: Recent progress. Helv. Phys. Acta 1996, 69, 570-581.

187. Erdmenger, J.; Osborn, H. Conserved currents and the energy momentum tensor in conformally invariant theories for general dimensions. Nucl. Phys. B 1997, 483, 431-474. [CrossRef]

188. Erdmenger, J. Conformally covariant differential operators: Properties and applications. Class. Quantum Gravity 1997, 14, 20612084. [CrossRef]

189. Deser, S. Closed form effective conformal anomaly actions in D > =4. Phys. Lett. B 2000, 479, 315-320. [CrossRef]

190. Bautista, T.; Benevides, A.; Dabholkar, A. Nonlocal Quantum Effective Actions in Weyl-Flat Spacetimes. J. High Energy Phys. 2018, 6, 055. [CrossRef]

191. Donoghue, J.F.; El-Menoufi, B.K. Covariant non-local action for massless QED and the curvature expansion. J. High Energy Phys. 2015, 10, 044. [CrossRef]

192. Benedetti, D.; Machado, P.F.; Saueressig, F. Taming perturbative divergences in asymptotically safe gravity. Nucl. Phys. B 2010, 824, 168-191. [CrossRef]

193. Stelle, K.S. Classical Gravity with Higher Derivatives. Class. Quantum Gravity 1978, 9, 353-371. [CrossRef]

194. Platania, A.; Wetterich, C. Non-perturbative unitarity and fictitious ghosts in quantum gravity. Phys. Lett. B 2020,811, 135911. [CrossRef]

195. Bonanno, A.; Denz, T.; Pawlowski, J.M.; Reichert, M. Reconstructing the graviton. arXiv 2021, arXiv:2102.02217.

196. Myers, R.C.; Simon, J.Z. Black Hole Thermodynamics in Lovelock Gravity. Phys. Rev. D 1988, 38, 2434-2444. [CrossRef]

197. Myers, R.C. Black holes in higher curvature gravity. In Black Holes, Gravitational Radiation and the Universe: Essays in Honor of C.V. Vishveshwara; Iyer, B.R., Bhawal, B., Eds.; Springer: Dordrecht, The Netherlands, 1998. [CrossRef]

198. Clunan, T.; Ross, S.F.; Smith, D.J. On Gauss-Bonnet black hole entropy. Class. Quantum Gravity 2004, 21, 3447-3458. [CrossRef]

199. Martin, J.; Ringeval, C.; Vennin, V. Encyclopædia Inflationaris. Phys. Dark Univ. 2014, 5, 75-235. [CrossRef]

200. Basile, I.; Platania, A. Cosmological $\alpha^{\prime}$-corrections from the functional renormalization group. J. High Energy Phys. 2020, $21,045$. [CrossRef] 
201. Basile, I.; Platania, A. String Tension between de Sitter vacua and Curvature Corrections. arXiv 2021, arXiv:2103.06276.

202. Percacci, R. An Introduction to Covariant Quantum Gravity and Asymptotic Safety. In 100 Years of General Relativity; World Scientific: Singapore, 2017; Volume 3. [CrossRef]

203. Reuter, M.; Saueressig, F. Quantum Gravity and the Functional Renormalization Group; Cambridge University Press: Cambridge, UK, 2019.

204. Pawlowski, J.M.; Reichert, M. Quantum gravity: A fluctuating point of view. arXiv 2020, arXiv:2007.10353.

205. Falls, K.; Ohta, N.; Percacci, R. Towards the determination of the dimension of the critical surface in asymptotically safe gravity. Phys. Lett. B 2020, 810, 135773. [CrossRef]

206. Barvinsky, A.O.; Vilkovisky, G.A. Beyond the Schwinger-Dewitt Technique: Converting Loops Into Trees and In-In Currents. Nucl. Phys. B 1987, 282, 163-188. [CrossRef]

207. Barvinsky, A.O.; Vilkovisky, G.A. Covariant perturbation theory. 2: Second order in the curvature. General algorithms. Nucl. Phys. B 1990, 333, 471-511. [CrossRef]

208. Barvinsky, A.O.; Vilkovisky, G.A. Covariant perturbation theory. 3: Spectral representations of the third order form-factors. Nucl. Phys. B 1990, 333, 512-524. [CrossRef]

209. Barvinsky, A.O.; Gusev, Y.V.; Zhytnikov, V.V.; Vilkovisky, G.A. Covariant perturbation theory. 4 . Third order in the curvature. arXiv 1993, arXiv:0911.1168.

210. Avramidi, I.G. The Nonlocal Structure of the One Loop Effective Action via Partial Summation of the Asymptotic Expansion. Phys. Lett. B 1990, 236, 443-449. [CrossRef]

211. Codello, A.; Zanusso, O. On the non-local heat kernel expansion. J. Math. Phys. 2013, 54, 013513. [CrossRef]

212. Ohta, N.; Rachwal, L. Effective action from the functional renormalization group. Eur. Phys. J. C 2020, 80, 877. [CrossRef]

213. Donnelly, W.; Giddings, S.B. Diffeomorphism-invariant observables and their nonlocal algebra. Phys. Rev. D 2016, 93, 024030; Erratum in 2016, 94, 029903. [CrossRef]

214. Rejzner, K. Effective quantum gravity observables and locally covariant QFT. Int. J. Mod. Phys. 2017, 1, 13-28. [CrossRef]

215. Donnelly, W.; Giddings, S.B. Observables, gravitational dressing, and obstructions to locality and subsystems. Phys. Rev. D 2016, 94, 104038. [CrossRef]

216. Klitgaard, N.; Loll, R. Introducing Quantum Ricci Curvature. Phys. Rev. D 2018, 97, 046008. [CrossRef]

217. Rudelius, T. Asymptotic Observables and the Swampland. arXiv 2021, arXiv:2106.09026.

218. Susskind, L.; Thorlacius, L.; Uglum, J. The Stretched horizon and black hole complementarity. Phys. Rev. D 1993, 48, $3743-3761$. [CrossRef] [PubMed]

219. Almheiri, A.; Marolf, D.; Polchinski, J.; Sully, J. Black Holes: Complementarity or Firewalls? J. High Energy Phys. $2013,2,062$. [CrossRef]

220. Giddings, S.B. Nonviolent nonlocality. Phys. Rev. D 2013, 88, 064023. [CrossRef]

221. Dvali, G.; Gomez, C.; Isermann, R.S.; Lüst, D.; Stieberger, S. Black hole formation and classicalization in ultra-Planckian 2 - N scattering. Nucl. Phys. B 2015, 893, 187-235. [CrossRef]

222. Keltner, L.; Tolley, A.J. UV properties of Galileons: Spectral Densities. arXiv 2015, arXiv:1502.05706.

223. Mann, R.B. Black Holes: Thermodynamics, Information, and Firewalls; Springer Briefs in Physics; Springer: Berlin, Germany, 2015. [CrossRef]

224. Modesto, L. Super-renormalizable Quantum Gravity. Phys. Rev. D 2012, 86, 044005. [CrossRef]

225. Modesto, L.; Rachwał, L.A. Nonlocal quantum gravity: A review. Int. J. Mod. Phys. D 2017, 26, 1730020. [CrossRef]

226. Buoninfante, L.; Lambiase, G.; Mazumdar, A. Ghost-free infinite derivative quantum field theory. Nucl. Phys. B 2019, $944,114646$. [CrossRef]

227. Buoninfante, L.; Koshelev, A.S.; Lambiase, G.; Mazumdar, A. Classical properties of non-local, ghost- and singularity-free gravity. J. Cosmol. Astropart. Phys. 2018, 9, 034. [CrossRef]

228. Giddings, S.B. Locality in quantum gravity and string theory. Phys. Rev. D 2006, 74, 106006. [CrossRef]

229. Buoninfante, L.; Di Filippo, F.; Mukohyama, S. On the assumptions leading to the information loss paradox. arXiv 2021, arXiv:2107.05662.

230. Veneziano, G. Scale factor duality for classical and quantum strings. Phys. Lett. B 1991, 265, 287-294. [CrossRef]

231. Meissner, K.; Veneziano, G. Symmetries of cosmological superstring vacua. Phys. Lett. B 1991, 267, 33-36. [CrossRef]

232. Meissner, K.A. Symmetries of higher order string gravity actions. Phys. Lett. B 1997, 392, 298-304. [CrossRef]

233. Hohm, O.; Zwiebach, B. T-duality Constraints on Higher Derivatives Revisited. J. High Energy Phys. 2016, 4, 101. [CrossRef]

234. Hohm, O.; Zwiebach, B. Non-perturbative de Sitter vacua via $\alpha^{\prime}$ corrections. Int. J. Mod. Phys. D 2019, 28, 1943002. [CrossRef]

235. Hohm, O.; Zwiebach, B. Duality invariant cosmology to all orders in $\alpha^{\prime}$. Phys. Rev. D 2019, 100, 126011, [CrossRef]

236. Held, A. Effective asymptotic safety and its predictive power: Gauge-Yukawa theories. Front. Phys. 2020, 8, 341. [CrossRef]

237. Eichhorn, A.; Pauly, M. Constraining power of asymptotic safety for scalar fields. Phys. Rev. D 2021, 103, 026006. [CrossRef]

238. Ali, P.; Eichhorn, A.; Pauly, M.; Scherer, M.M. Constraints on discrete global symmetries in quantum gravity. J. High Energy Phys. 2021, 5, 036. [CrossRef]

239. Banks, T.; Dixon, L.J. Constraints on String Vacua with Space-Time Supersymmetry. Nucl. Phys. B 1988, 307, 93-108. [CrossRef]

240. Kallosh, R.; Linde, A.D.; Linde, D.A.; Susskind, L. Gravity and global symmetries. Phys. Rev. D 1995, 52, 912-935. [CrossRef]

241. McNamara, J.; Vafa, C. Cobordism Classes and the Swampland. arXiv 2019, arXiv:1909.10355. 